\title{
Vertebrates in the "Palude di Torre Flavia" Special Protection Area (Lazio, central Italy): an updated checklist
}

\author{
Corrado Battisti ${ }^{1,2 *}$, Michele Cento ${ }^{2}$, Fulvio Fraticelli ${ }^{2}$, Steven Hueting ${ }^{2}$, \\ Sergio Muratore ${ }^{2}$
}

\begin{abstract}
Here we present the checklist of the vertebrates of the "Palude di Torre Flavia", a protected area in Tyrrhenian central Italy (Special Protection Area according to the Directive 2009/147/EC). To draw up the checklist, we collated all the records found in the literature, in private collections, and in the Ornitho.it website database, as well as sporadic historical observations. We obtained evidence documented between 1981 and 2020 for 291 taxa of which 259 native, 26 allochthonous and 6 domesticated species in 5 classes: 5 actinopterygians (4 native species and 1 allochtonous), 2 amphibians, 20 reptiles (11 native species and 9 allochthonous; the last being all freshwater terrapins), 244 birds (including 14 non-native taxa and 6 domestic forms) and 20 mammals (including 2 allochthonous). Forty-three species are listed as of conservation concern on a national scale. The area has shown to be an important biodiversity hotspot, and a major stopover site for migrating birds. Further research should be focused on some still poorly investigated taxonomic groups, in particular: Actinopterygii, Amphibia Salamandridae, Carnivora Mustelidae, and Chiroptera.
\end{abstract}

Key words: Actinopterygii, Amphibia, Aves, Mammalia, remnan wetlands, Reptilia.

Riassunto - Vertebrati nella Zona di Protezione Speciale "Palude di Torre Flavia" (Lazio, Italia centrale): una checklist aggiornata.

Riportiamo la checklist dei vertebrati della "Palude di Torre Flavia", un'area protetta del litorale tirrenico dell'Italia centrale (Zona di Protezione Speciale, Dir. "Uccelli" 147/2009/CE). La lista è stata ottenuta da una revisione di dati originali, bibliografici e da collezioni naturalistiche private, includendo anche occasionali dati storici. Nell'area protetta sono state ottenute evidenze di presenza per il periodo 1981-2020 per 291 taxa (259 autoctoni, 26 alloctoni e 6 forme domestiche): 5 specie di attinopterigi (4 specie autoctone e 1 alloctona), 2 di anfibi, 20 di rettili (11 specie autoctone e 9 alloctone, queste ultime tutte specie di tartaru-

\footnotetext{
'Torre Flavia' LTER (Long Term Ecological Research) Station, Città Metropolitana di Roma, Protected Areas Service, Via G. Ribotta 41, 00144 Roma, Italia.

${ }^{2}$ SROPU (Stazione Romana Osservazione e Protezione Uccelli), Piazza Margana 40, 00186 Roma, Italia.

E-mail: michi.100@libero.it; f fraticelli@hotmail.com; gshueting@gmail.com; sergio_muratore@yahoo.it
}

* Corresponding author: c.battisti@cittametropolitanaroma.gov.it

(C) 2021 Corrado Battisti, Michele Cento, Fulvio Fraticelli, Steven Hueting, Sergio Muratore

Received for publication: 7 December 2020

Accepted for publication: 17 March 2021

Online publication: 3 May 2021 ghe di acqua dolce in commercio), 244 di uccelli (tra cui 14 alloctone e 6 forme domestiche) e 20 di mammiferi (incluse 2 non native). La Palude di Torre Flavia si conferma come un hot-spot di diversità biologica, soprattutto per gli uccelli $(86.5 \%$ sul totale dei Vertebrati autoctoni), in gran parte migratori, per i quali essa costituisce un importante sito di sosta. Alcuni gruppi tassonomici (es., Actinopterygii, Amphibia Salamandridae, Carnivora Mustelidae e Chiroptera) richiedono un supplemento di indagine.

Parole chiave: Actinopterygii, Amphibia, Aves, Mammalia, palude residuale, Reptilia.

\section{INTRODUCTION}

Biological checklists provide an important tool in assessing the value of a protected area, and their compilation is a strategic step in measuring biodiversity, allowing the description of qualitative and quantitative trends of species composition and richness over time. Ckecklists can suggest priority targets in research and conservation strategies even at the level of the single species (Sutherland, 2008). Examples of faunal checklists for single protected areas in central Italy are available both for all vertebrates (e.g., Crucitti et al., 2013; Battisti, 2014a; Crucitti et al., 2019), and for single taxonomic groups (e.g., Romano et al., 2007; Marini et al., 2014; Mori et al., 2014).

The Mediterranean coastal wetlands are ecosystems of great environmental interest, with a high biological diversity and carriers of many ecological benefits (Mediterranean Wetlands Observatory, 2012, 2018; Davidson et al., 2018). Nevertheless, they are now extremely fragmented and of small extension, if compared to how they were some decades ago (Britton \& Crivelli, 1993; Blondel \& Aronson, 1999).

In central Tyrrhenian Italy, most of the originally existing wetlands has been reclaimed during the $19^{\text {th }}$ and early $20^{\text {th }}$ centuries, causing a significant loss of their biodiversity. Other major factors of human impact are urbanisation and the construction of road networks, which produce devastating consequences on wetlands, in particular, by triggering habitat fragmentation and degradation, diffusion of nonnative species, pollution, water stress and intense poaching, especially on birds (Battisti et al., 2008; see historical information in Rotondi, 1962). Remnant wetlands along the coast north of Rome form an 'archipelago' of small islands of biodiversity (e.g., Fraticelli et al., 2016). One of these is the 'Palude di Torre Flavia' (Battisti, 2006a). Hunting in 
this area was carried out since paleo-historical times (see Fatucci \& Cerilli, 2015), and faunal records are available thanks to hunting reports that were drawn up for over a century (e.g., Patrizi-Montoro, 1909; Di Tucci \& Chigi, 1937; Pagliari, 1952), until the 1970s and 1980s (Pratesi, 2006). In 1997, this wetland was declared a Natural Monument, and subsequently it was included in the European network of Special Protection Areas (EU 147/2009 'Birds' Directive: Battisti \& Buccomino, 1999). With the establishment of the protected area, most of the research was directed to the knowledge of flora, vegetation, fauna and other ecosystem components (Battisti, 2006a), and partly included in the activities of the local research station settled by LTER - Long Term Ecological Research (Bertoni, 2012). More specifically, this site has been the subject of studies on microphytes (diatoms: Della Bella et al., 2006, 2007), macrophytes (Guidi, 2006; Buccomino \& Leporatti, 2009; Nimis et al., 2020; specific research and reviews: Lucchese, 1996; Aglitti et al., 2006; Fanelli, 2006; Santoro et al., 2012; Acosta et al., 2013; Del Vecchio et al. 2018; Ioni et al., 2020; floristic notes: Bartolucci \& Iocchi, 2007; Anzalone et al., 2010; Fanelli et al., 2011; Fabrini, 2019), microfaunal benthos (Foraminifera, Ostracoda and others: Nicoletti et al., 2006; Raffi et al., 2018; Di Bella et al., 2020), freshwater macro-benthos (Gramegna et al., 2006), Crustacea Amphipoda (Iannilli et al., 2018), non-native Crustacea (Chiesa, 2006; Chiesa et al., 2006; Scalici et al., 2009; Scalici et al., 2010), freshwater and terrestrial Mollusca (Minganti \& Zocchi, 2006), marine Mollusca (Pessolano et al., 2006; notes: e.g. Amati et al., 2019; Amati et al., 2020; Battisti, 2020; Giannuzzi-Savelli et al., 2020), Chilopoda (Zapparoli, 2006), Hymenoptera (Di Giovanni \& Reshchikov, 2016), Odonata (Dell'Anna \& Avellinese, 2006), Coleoptera (Carpaneto et al., 2006; notes: De Giovanni, 2015), Hemyptera (notes: Dioli, 2010), Lepidoptera (Zilli, 2006).

Regarding Vertebrata, species lists have been compiled for Actinopterygii (Celauro, 2006), Amphibia and Reptilia (Pitzalis \& Bologna, 2006), Aves (Battisti \& Sorace, 2006) and Mammalia (Amori et al., 2006). More recently, non-standardized research and surveys (often unpublished) provided new and useful information on the species richness of the area.

To obtain the checklists for each vertebrate group, we have carried out a review of the available literature, of the catalogue of the Ruspoli ornithological collection in Cerveteri and of unpublished data collected by a large number of volunteers and researchers, which investigated the area over two decades. We also provided detailed information on the status of single species at local scale, aiming to deliver useful tools for future conservation strategies and actions promoted by the Park Agency managing this area.

\section{MATERIALS AND METHODS}

\section{Study area}

The study area includes the whole Natural Monument "Palude di Torre Flavia" (municipalities of Cerveteri and Ladispoli; Lazio, central Italy; $41^{\circ} 58^{\prime} \mathrm{N}, 12^{\circ} 03^{\prime} \mathrm{E}$ ), a small wetland area (40 ha) on the Tyrrhenian coast (Special Protection Area according to the Directive 2009/147/
EC 'Birds'; code IT6030020), relict of a larger wetland drained and transformed by land reclamation (Battisti, 2006a; Battisti et al., 2006) (Figs. 1 and 2).

At a landscape scale, this area represents a remnant fragment of wetland inside an agricultural and urbanized matrix. At a local scale, it shows a seminatural heterogeneity with Phragmites australis reed-beds and ponds used for fish farming from 1938 (mainly managing stocks of Anguilla anguilla and three species of mullets, Mugil cephalus, Chelon saliens, Chelon ramada; Moccia, 2006). From 2004, activities of fish stock management like flooding, reedbed mowing and burning (Battisti et al., 2009a, $2009 b)$ were completely abandoned. Near the reedbeds there are flooded meadows with Carex hirta, Juncus acutus and Cyperaceae corresponding to the Juncetalia maritimi habitat type according to the "Habitat" Directive 92/43/EC (Guidi, 2006; Fanelli \& Bianco, 2007). The water flooding the wetland is mainly of meteoric and sea
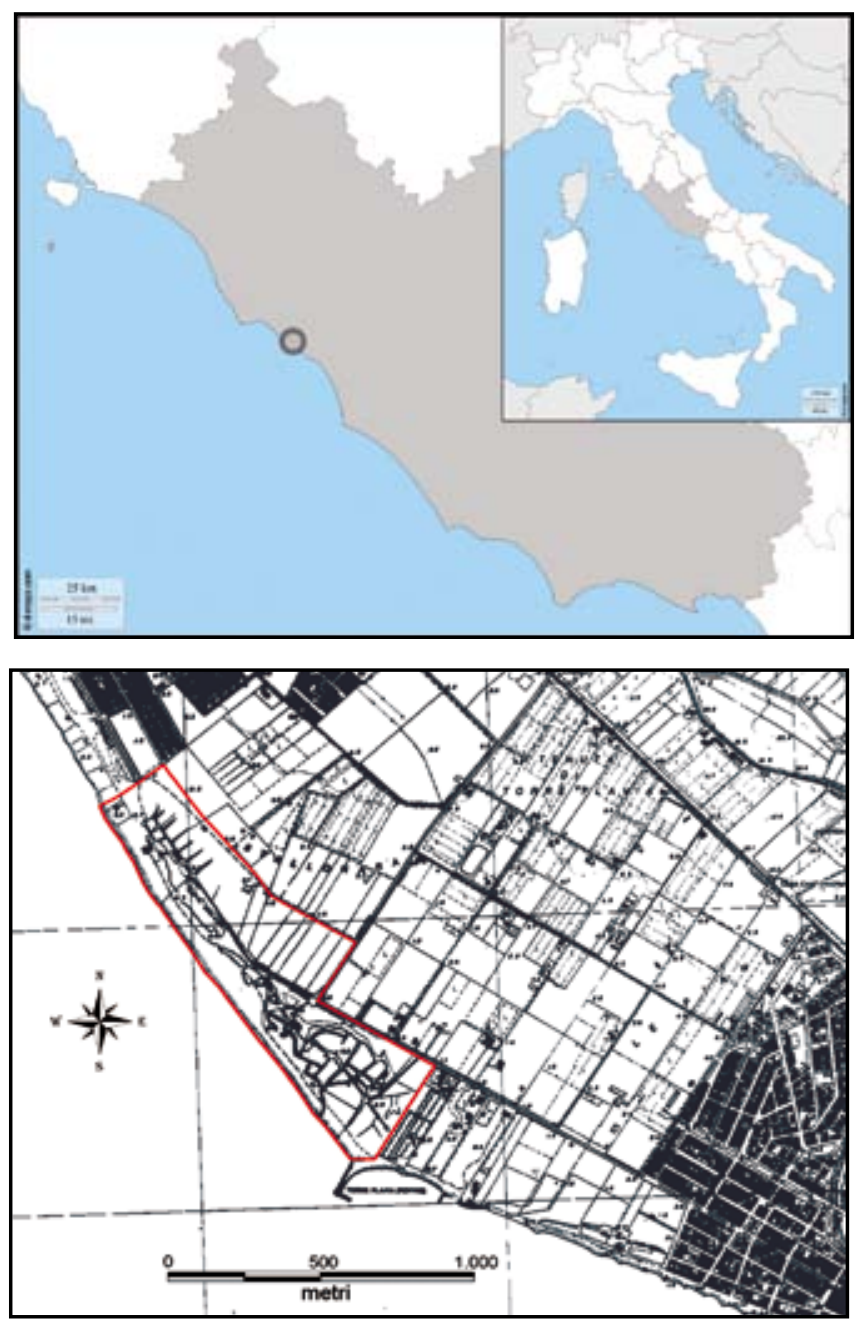

Fig. 1 - Map of the study area. Top: geographical location at regional and national level. Bottom: "Palude di Torre Flavia" Natural Monument near the urbanized area of Ladispoli (Rome, central Italy; perimeter of the protected area in red; source: Regione Lazio - Technical Map having grid with 1x1 km-wide units). / Mappa dell'area di studio. In alto: localizzazione geografica a scala nazionale e regionale. In basso: Monumento naturale "Palude di Torre Flavia", limitrofo alla città di Ladispoli (Roma, Italia centrale; fonte: Regione Lazio - Carta tecnica con griglia con unità di 1x1 km di larghezza). 


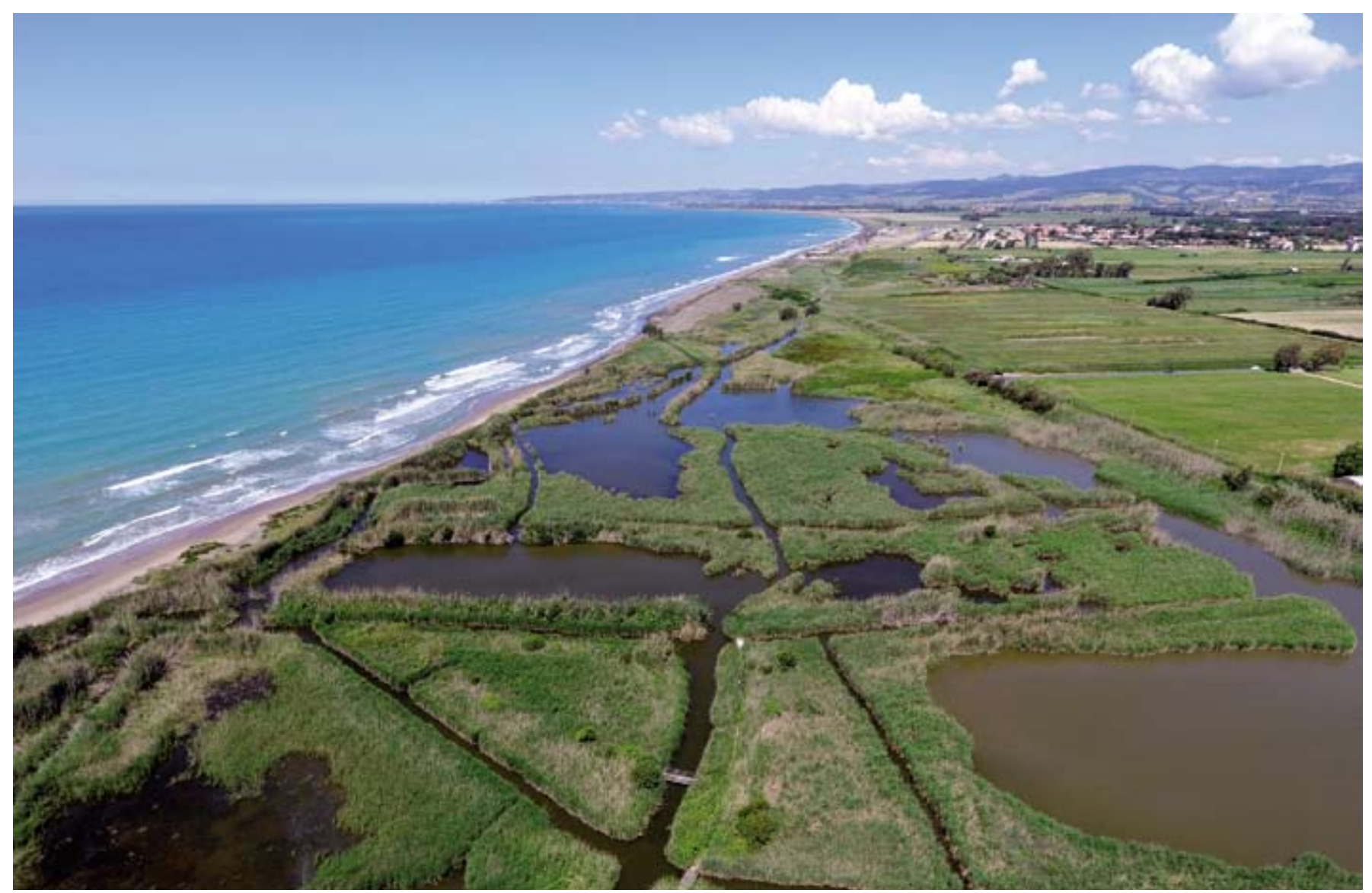

Fig. 2 - The "Palude di Torre Flavia" in an aerial shot taken by a drone. / La "Palude di Torre Flavia" in una ripresa aerea realizzata da un drone. (Photo:/ Foto:L. Cicillini).

storm origin (Battisti, 2006a; Battisti et al., 2008). Along the coastline patches of the EU Habitat type "Embryonic shifting dunes" (code 2110) are present (Guidi, 2006; Ioni et al., 2020). Climate is xeric-meso-Mediterranean (Blasi \& Michetti, 2005). For details on chemistry and water quality: Sabia et al. (2018); for morphodynamics of the coastal landscape, see Raffi et al. (2018), Davoli et al. (2019).

\section{METHODS}

We collated data from unpublished field notes, private collections, bibliographic references, atlases and datasets of vertebrate species occurring in the Torre Flavia wetland and the proximate areas (considering a buffer zone about $1 \mathrm{~km}$ deep) from 1981 to 2020 , obtaining a checklist for each taxonomic group (Actinopterygii, Amphibia, Reptilia, Aves and Mammalia). When available, we also added some historical data before 1981.

Bibliographic information was first obtained from faunal lists dedicated to single (Actinopterygii: Celauro, 2006; Moccia, 2006; Aves: Battisti \& Sorace, 2006; Mammalia: Amori et al., 2006) or traditionally grouped (Amphibia and Reptilia: Pitzalis \& Bologna, 2006; Pitzalis, 2007) classes of vertebrates. Further information was obtained from other sources (see Tables
1-5), including regional and provincial atlases (Bologna et al., 2000, 2007; Amori et al., 2009; Brunelli et $a l ., 2011)$. For Aves in particular, we used unpublished original information, the citizen science web platform Ornitho.it (Updated: 31 October 2020; permission requested), and the record database of the local ringing station.

For each species we reported its conservation status at the national level. For the threat category we followed Rondinini et al. (2013), updated for birds by Gustin et al. (2019).

For birds, the phenological categories are those recommended by Baccetti et al. (2019). We have considered a bird species as regularly present or nesting if observed in at least 9 of the last 10 years, irregularly present or nesting if observed in more than 5 and less than 9 of the last 10 years, and accidental when observed no more than five times. We also considered as wintering those species present at least during part of the December-January period (see Brichetti \& Fracasso, 2015).

Concerning the taxonomic treatment, we followed the recently adopted nomenclatures for Italian vertebrates (Actinopterygii: Lorenzoni et al., 2019; Amphibia and Reptilia: Speybroeck et al., 2020; Aves: Baccetti et al., 2019; Mammalia: Boitani et al., 2003; Amori et al., 2008; Lanza, 2012, updated with the last revision of Loy et al., 2019). 


\section{RESULTS AND DISCUSSION}

\section{Actinopterygii}

There is confirmed evidence for at least five species (four native and one non-native, Tab. 1). The record of Pseudorasbora parva (Temminck \& Schlegel, 1846) reported in Celauro (2006) has not been confirmed by a morphological diagnosis (S. Zerunian, pers. comm.) and not included in the checklist. Also, the specific attributions for Sarmarutilus rubilio Bonaparte, 1837, reported in Cealuro (2006) and Scardinius erythrophthalmus (Linnaeus, 1758) (P. Tinazzo, pers. comm.) require confirmation and have not been included in the checklist: the second (and perhaps also the first), if confirmed, have been probably introduced during the water pumping carried out by the Land Reclamation Agency ('Consorzio di Bonifica 'Tevere e Agro Romano'). A careful investigation to verify the possible presence of other species introduced through the pipes draining water from the Tiber to the wetland, could be deciding.

The prevalent fish biomass consists of three euryhaline Mugilidae species, actively transported in autumn from the sea to the wetland between 1938 and 2004 as part of the fish farming activity. Fish farming was interrupted in 2004 although occasional translocations have been carried out in the following years (Battisti \& Sorace, 2005; Moccia, 2006 and pers. obs.). Ano- ther species locally occurs with a high biomass: Gambusia hoolbrooki (Girard, 1859), an allochthonous species also inhabiting brackish waters, introduced as a malaria control agent during the land reclamation occurred in the first decades of the $20^{\text {th }}$ century ( $\mathrm{Ga}$ chelin et al., 2018). This species is considered one of the most invasive species worldwide (Webb \& Joss, 1997; Vannini et al., 2018). However, due to its abundance, ease to capture and appeal for children, this species has recently been proposed as 'experiential key species' for local conservation education strategies (Battisti, 2016; Bertolino, 2016; Battisti et al., 2018b). Anguilla anguilla (Linnaeus, 1758) turns out to be the species classified in the highest conservation concern category (Critically endangered; Rondinini et al., 2013).

Fish farming created the conditions for the establishment of a trophic network including many ichthyophagous species, mainly birds. The abandonment of this activity made a new management plan for the ichthyofauna necessary, with periodic removal and translocation of mullets in excess (Battisti et al., $2020 \mathrm{c})$. Furthermore, with the abandonment of fish farming management activities from 2004, reedbeds were no longer mown. This caused a structural shift of the wetland vegetation that triggered significant faunal changes (e.g., in bird assemblages: Battisti et al., 2020a).

Tab. 1 - Check list of ray-finned fishes (Actinopterygii) occurring in the Torre Flavia wetland. Taxonomic treatment after Lorenzoni et al. (2019). Inclusion in global or Italian IUCN Red Lists is reported (Rondinini et al., 2013; Relini et al., 2017). CR = Critically Endangered; LC = Least Concern; NE = Not Evaluated. / Check list degli attinopterigi (Actinopterygii) presenti nella Palude di Torre Flavia. Trattamento tassonomico secondo Lorenzoni et al. (2019). Viene riportata l'inclusione nelle Liste Rosse IUCN globale o italiana (Rondinini et al., 2013; Relini et al., 2017). CR = In Pericolo Critico; LC = Minor preoccupazione; NE = Non Valutata.

\begin{tabular}{|c|c|c|}
\hline Taxa & Red List & $\begin{array}{l}\text { Notes, bibliographic references (in chronological order) and personal observations } \\
\text { (nr. of individuals, date, names of observers) }\end{array}$ \\
\hline \multicolumn{3}{|l|}{ ACTINOPTERYGII } \\
\hline \multicolumn{3}{|l|}{ Anguillidae } \\
\hline Anguilla anguilla (Linnaeus, 1758) & $\mathrm{CR}$ & Celauro, 2006; Battisti \& Zocchi, 2018 and pers. obs. \\
\hline Mugil cephalus Linnaeus, 1758 & $\mathrm{LC}$ & $\begin{array}{l}\text { Mainly translocated from neighbouring areas for fish farming; Battisti \& Sorace, 2005; } \\
\text { Celauro, 2006; Moccia, 2006; Battisti, 2016; Battisti \& Zocchi, } 2018 \text { and pers. obs. }\end{array}$ \\
\hline Chelon ramada (Risso, 1827) & $\mathrm{LC}$ & $\begin{array}{l}\text { Mainly translocated from neighbouring areas for fish farming; Battisti \& Sorace, 2005; } \\
\text { Celauro, 2006; Moccia, 2006; Battisti, 2016; Battisti \& Zocchi, } 2018 \text { and pers. obs. }\end{array}$ \\
\hline Chelon saliens (Risso, 1810) & $\mathrm{LC}$ & $\begin{array}{l}\text { Mainly translocated from neighbouring areas for fish farming; Battisti \& Sorace, 2005; } \\
\text { Celauro, 2006, Moccia, 2006; Battisti \& Zocchi, } 2018 \text { and pers. obs. }\end{array}$ \\
\hline \multicolumn{3}{|l|}{ Poecilidae } \\
\hline Gambusia hoolbrooki Girard, 1859 & $\mathrm{NE}$ & $\begin{array}{l}\text { Historically introduced; Celauro, 2006; Amori \& Battisti, 2008; Battisti, 2016; Bertolino, } \\
\text { 2016; Battisti et al., 2018b; Battisti \& Zocchi, } 2018 \text { and pers. obs. }\end{array}$ \\
\hline
\end{tabular}




\section{Amphibia}

We were able to confirm data for only two species of low conservation concern (Tab. 2). The only two species of Caudata Salamandridae - Triturus carnifex (Laurenti, 1768 ) and Lissotriton vulgaris (Linnaeus, 1758) - reported as locally occurring in previous checklists (Pitzalis \& Bologna, 2006; Pitzalis, 2007), have never been found in successive years: therefore, we excluded them from the checklist. For the same reason, among Anura Bufonidae, we excluded Bufotes viridis (Laurenti, 1768): for this species further investigation is anyway required. It is likely that the richness and abundance of amphibians is limited by the presence of brackish water and fish, as well as by the direct or indirect impact of alien species (Amori \& Battisti, 2008; e.g., freshwater turtles and the non-native crayfish Procambarus clarkii Girard, 1852; see Ficetola et al., 2011). Many individuals of Bufo bufo (Linnaeus, 1758 ) are run over by cars along the roads along the border of the protected area: to mitigate this threat, some defragmentation interventions have been locally implemented (Scoccianti, 2006).

\section{Reptilia}

Reptiles are present with 11 native species (2 Testudines, 5 Squamata, 4 Serpentes) and 9 allochthonous (all freshwater terrapins, Testudines; Tab. 3). Among the native species, two are classified as Endangered (both Testudines): Emys orbicularis (Linnaeus, 1758) and Caretta caretta (Linnaeus, 1758). In July 2020 a nest of this last species has been translocated from a neighboring site (Palo Laziale, about $2 \mathrm{~km}$ far away) to the northern beach of Torre Flavia protected area (Tab. 3; two nesting attempts occurred in this area during the same period; M. Perchinelli, pers. obs.). Considering the northward shift of $\mathrm{Ca}$ retta caretta nesting that has taken place in recent years (L. Marini, TARTA Lazio, pers. comm.; Mingozzi et al., 2007; Bentivegna et al., 2010), the beach of Torre Flavia could be considered potentially suitable for this chelonian. The presence of Anguis veronensis Pollini, 1818, reported in Pitzalis \& Bologna (2006) and Pitzalis (2007), needs to be confirmed and has not been included in the checklist.
The abundance of the two species of the genus Natrix is likely the result of the great availability of fish. Along embryonic dunes, an abundant population of Podarcis siculus (Rafinesque-Schmaltz, 1810) also occurs. Furthermore, the study area hosts a high number of alien terrapins, probably illegally introduced on several occasions by people. We have records of naturalization (juv. observed) for two species: Trachemys scripta (Thunberg in Schoepff, 1792) and Mauremys sinensis (Gray, 1834) (see Ferri et al., 2019, 2020; Di Blasio et al., in press).

\section{Aves}

We found data for 224 autochtonous species (36 of them, $16 \%$, breed in the study area and six probably breed), 14 non-native taxa and 6 domestic forms (Tab. 4). Three species - Puffinus lherminieri Lesson, 1839, Garrulus glandarius (Linnaeus, 1758), Passer hispaniolensis (Temminck, 1820) - have not been confirmed. Also, two historical observations for Aythya marila (Linnaeus, 1761) (in 1962-1963: G. Lepri Angelini, pers. comm.) and Syrrhaptes paradoxus (Pallas, 1773) (Patrizi Montoro, 1909; Brunelli \& Fraticelli, 2010), were carried out outside the time range considered in this study and have not been included.

Forty-three species are of conservation concern according to the Italian Red List (Gustin et al., 2019): one species is Regionally Extinct, five are Critically Endangered, 15 are Endangered and 22 are Vulnerable. Sixty-seven species are included in the annex I of the EU 147/2009 'Birds' Directive. Sixty-seven species are of conservation concern according to BirdLife international (BirdLife International, 2017): 13 of global conservation concern (SPEC 1), 17 of European conservation concern, concentrated in Europe (SPEC 2), 37 of European conservation concern, not concentrated in Europe (SPEC 3). Most of them are migratory birds.

Some other species were recorded immediately outside the study area and are not included in the checklist: for example, Ciconia nigra (Linnaeus, 1758) (2 ind., 18 Oct. 2012, M. Cento, A. Manganaro, pers. obs.; historical

Tab. 2 - Check list of amphibians (Amphibia) occurring in the Torre Flavia wetland. Taxonomic treatment after Lanza et al. (2007) and Speybroeck et al. (2020). Inclusion in global or Italian IUCN Red Lists is reported (Rondinini et al., 2013; LC = Least Concern). / Check list degli anfibi (Amphibia) presenti nella Palude di Torre Flavia. Trattamento tassonomico secondo Lanza et al (2007) e Speybroeck et al. (2020). Viene riportata l'inclusione nelle Liste Rosse IUCN globale o italiana (Rondinini et al., 2013; LC = Minor Preoccupazione).

\begin{tabular}{|c|c|c|}
\hline Taxa & Red List & $\begin{array}{l}\text { Notes, bibliographic references (in chronological order) and personal } \\
\text { observations (nr. of individuals, date, names of observers) }\end{array}$ \\
\hline \multicolumn{3}{|l|}{ AMPHIBIA } \\
\hline \multicolumn{3}{|l|}{ Bufonidae } \\
\hline Bufo bufo (Linnaeus, 1758) & $\mathrm{LC}$ & $\begin{array}{l}\text { Pitzalis \& Bologna, 2006; Pitzalis, 2007; Battisti, 2016; Battisti et al., 2018b; Battisti \& } \\
\text { Zocchi, 2018; C. Battisti, pers. obs. }\end{array}$ \\
\hline
\end{tabular}


records: 1962-1963: G. Lepri Angelini, pers. comm.) and Limnodromus scolopaceus (Furbara: Fraticelli, 1998). Although quantitative data on species density, frequency and diversity have been obtained with standardized methods (point counts, linear transects, mapping techniques, local atlases and photo-trapping), a good number of species, especially accidental ones, results from occasional observations by birdwatchers and professional ornithologists and from the local ringing station database (Sorace et al., 2001, 2003, 2006, 2010, 2015).

Tab. 3 - Check list of reptiles (Reptilia) occurring in the Torre Flavia wetland. Taxonomic treatment after Corti et al. (2011) and Speybroeck et al. (2020). Inclusion in global or Italian IUCN Red Lists is reported (Rondinini et al., 2013: EN = Endangered; LC = Least Concern). / Check list dei rettili (Reptilia) presenti nella Palude di Torre Flavia. Trattamento tassonomico secondo Corti et al. (2011) e Speybroeck et al. (2020). Viene riportata l'inclusione nelle Liste Rosse IUCN globale o italiana (Rondinini et al., 2013: EN = in Pericolo; LC = Minor Preoccupazione).

\begin{tabular}{|c|c|c|}
\hline Taxa & Red list & $\begin{array}{l}\text { Notes, bibliographic references (in chronological order) and personal } \\
\text { observations (nr. of individuals, date, names of observers) }\end{array}$ \\
\hline \multicolumn{3}{|l|}{ REPTILIA } \\
\hline \multicolumn{3}{|l|}{ Emydidae } \\
\hline Emys orbicularis (Linnaeus, 1758) & EN & $\begin{array}{l}\text { Pitzalis \& Bologna, 2006; Pitzalis, 2007; C. Battisti, F. Causarano, R. Santoro } \\
\text { pers. obs. }\end{array}$ \\
\hline Caretta caretta (Linnaeus, 1758) & EN & $\begin{array}{l}\text { Pitzalis \& Bologna, 2006; Pitzalis, 2007; L. Marini, C. Battisti, pers. obs.; } \\
\text { until } 2020 \text { only beached specimens. Nest translocated in July } 2020 \text { (91 eggs } \\
\text { laid; } 68 \text { hatched 25.9.2020) }\end{array}$ \\
\hline \multicolumn{3}{|l|}{ SQUAMATA } \\
\hline \multicolumn{3}{|l|}{ Scincidae } \\
\hline \multicolumn{3}{|l|}{ Lacertidae } \\
\hline Lacerta bilineata Daudin, 1802 & $\mathrm{LC}$ & $\begin{array}{l}\text { Pitzalis \& Bologna, 2006; Pitzalis, 2007; Battisti \& Zocchi, 2018; C. Battisti } \\
\text { and M. Bologna, pers. obs. }\end{array}$ \\
\hline Podarcis muralis (Laurenti, 1768) & $\mathrm{LC}$ & $\begin{array}{l}\text { Pitzalis \& Bologna, 2006; Pitzalis, 2007; Battisti \& Zocchi, 2018; C. Battisti, } \\
\text { pers. obs. }\end{array}$ \\
\hline Podarcis siculus (Rafinesque-Schmaltz, 1810) & $\mathrm{LC}$ & $\begin{array}{l}\text { Pitzalis \& Bologna, 2006; Pitzalis, 2007; Battisti \& Zocchi, 2018; C. Battisti } \\
\text { and M. Bologna, pers. obs. }\end{array}$ \\
\hline \multicolumn{3}{|l|}{ SERPENTES } \\
\hline \multicolumn{3}{|l|}{ Colubridae } \\
\hline Hierophis viridiflavus (Lacépède, 1789) & $\mathrm{LC}$ & Pitzalis \& Bologna, 2006; Pitzalis, 2007; C. Battisti, pers. obs. \\
\hline \multicolumn{3}{|l|}{ Kinosternidae } \\
\hline $\begin{array}{l}\text { Kinosternon subrubrum subrubrum (Bonnaterre, 1789) } \\
\text { vel Sternotherus odoratus (Latreille, 1801) }\end{array}$ & & $\begin{array}{l}\text { First record of free-ranging individuals of this family for Europe; Ferri et al., } \\
2019,2020,2021\end{array}$ \\
\hline \multicolumn{3}{|l|}{ Emydidae } \\
\hline Graptemys pseudogeographica (Gray, 1831) & & $\begin{array}{l}\text { Recorded in Lazio (Scalera \& Montinaro, 2014): both ssp. pseudogeographica } \\
\text { (Gray, 1831) and ssp. kohni (Baur, 1890); Ferri et al., 2019, } 2020\end{array}$ \\
\hline Pseudemys concinna concinna (Le Conte, 1830) & & $\begin{array}{l}\text { Recorded at genus level for Lazio (Scalera \& Montinaro, 2014); first record of } \\
\text { free-ranging individuals in Italy; Ferri et al., 2019, } 2020\end{array}$ \\
\hline Pseudemys nelsoni Carr, 1938 & & Ferri et al., 2019, 2020 \\
\hline
\end{tabular}




\begin{tabular}{|l|l|l|}
\hline Taxa & Red list & $\begin{array}{l}\text { Notes, bibliographic references (in chronological order) and personal } \\
\text { observations (nr. of individuals, date, names of observers) }\end{array}$ \\
\hline Trachemys scripta (Thunberg in Schoepff, 1792) & $\begin{array}{l}\text { Ssp. elegans (Wied, 1839): first record of juveniles born in the wild for Lazio; } \\
\text { ssp. scripta (Schoepff, 1792): first record of juveniles born in the wild for } \\
\text { Lazio; in the area, also the ssp. troostii Seidel, 2002 and hybrids occur; Ferri } \\
\text { et al., 2020; see also: Pitzalis \& Bologna, 2006; Pitzalis, 2007; Amori \& } \\
\text { Battisti, 2008; Battisti \& Zocchi, 2018; Grillo et al., 2020 and pers. obs. (first } \\
\text { record: C. Battisti, E. De Angelis, C. Galimberti, N. Trucchia; 2004). }\end{array}$ \\
\hline Trachemys venusta (Gray, 1855) & First record for Lazio, 2020; V. Ferri \& R. Santoro, pers. obs. \\
\hline Geoemydidae & & $\begin{array}{l}\text { Already recorded at genus level for Lazio (Scalera \& Montinaro, 2014); first } \\
\text { record of free ranging individuals in Italy; first record of juv., for Europe } \\
\text { (Ferri } \text { et al., 2019, 2020, 2021) }\end{array}$ \\
\hline Mauremys sinensis (Gray, 1834) & \\
\hline Pelomedusidae & $\begin{array}{l}\text { First record of free-ranging individuals for Europe; Ferri et al., 2019, 2020, } \\
\text { 2021 }\end{array}$ \\
\hline Pelomedusa olivacea (Schweigger, 1812) & & $\begin{array}{l}\text { First record of free-ranging individuals in Torre Flavia; NOGRA-Nucleo } \\
\text { Operativo Guardie Zoofile-Ambientali, pers. obs. }\end{array}$ \\
\hline Chelydridae & & \\
\hline Chelydra serpentina (Linnaeus, 1758) & & \\
\hline
\end{tabular}

Tab. 4 - Check list of the birds (Aves) occurring in the Torre Flavia wetland. Taxonomic treatment after Baccetti et al., 2019. Phenology: $\mathrm{S}=$ Sedentary; $\mathrm{B}=$ Breeding; $\mathrm{M}=$ Migratory; reg = regular; irr = irregular; $\mathrm{W}=$ Wintering; $\mathrm{S}=$ Summer visitor; $\mathrm{A}=$ Accidental (see Methods for details). Inclusion in global or Italian IUCN Red Lists is also reported (Gustin et al., 2019: RE = Regionally Extinct; CR = Critically Endangered; $\mathrm{EN}=$ Endangered; VU = Vulnerable; the Least Concern, LC, category is omitted). / Check list degli uccelli (Aves) presenti nella Palude di Torre Flavia. Trattamento tassonomico da Baccetti et al. (2019). Fenologia: $\mathrm{S}=$ sedentaria; $\mathrm{B}=$ nidificante; $\mathrm{M}=$ migratrice; reg = regolare; $\mathrm{irr}=$ irregolare; $\mathrm{W}=$ svernante; $\mathrm{S}=$ estivante; $\mathrm{A}=$ accidentale (vedi Metodi per i dettagli). Viene anche segnalata l'inclusione nelle Liste Rosse IUCN globale o italiana (Gustin et al., 2019: $\mathrm{RE}=$ estinto a livello regionale; $\mathrm{CR}=$ in Pericolo Critico; $\mathrm{EN}=$ in Pericolo; VU = Vulnerabile; la categoria Minor Preoccupazione, LC, è omessa).

\begin{tabular}{|c|c|c|c|}
\hline Taxa & Phenology & Red List & $\begin{array}{l}\text { Notes, bibliographic references (in chronological order) and personal } \\
\text { observations (nr. of individuals, date, names of observers) }\end{array}$ \\
\hline \multicolumn{4}{|l|}{ AVES } \\
\hline \multicolumn{4}{|l|}{ GALLIFORMES } \\
\hline \multicolumn{4}{|l|}{ Phasianidae } \\
\hline Coturnix coturnix (Linnaeus, 1758) & M irr, B irr & & $\begin{array}{l}\text { Sorace et al., 2001; Battisti, 2006b; Battisti \& Sorace, 2006; Sorace et al., } \\
\text { 2006; Battisti et al., 2020b }\end{array}$ \\
\hline \multicolumn{4}{|l|}{ ANSERIFORMES } \\
\hline \multicolumn{4}{|l|}{ Anatidae } \\
\hline Anser anser (Linnaeus, 1758) & A & & $\begin{array}{l}\text { Max } 55 \text { ind., 7.2.2004, R. Gildi, V. Dundee; } 25 \text { ind., 11.2.2004, M. Biondi; } \\
\text { Battisti \& Sorace, 2006; Battisti } \text { et al., 2012a; Battisti, 2014b }\end{array}$ \\
\hline Anser albifrons (Scopoli, 1769) & A & & Molajoli \& Catoni, 2001: 102 ind.; Battisti \& Sorace, 2006 \\
\hline Melanitta fusca (Linnaeus, 1758) & A & & 1 ind., 28.12.2013, A. Manganaro; 1 ind., 1.3.2017, S. Di Carlo \\
\hline Mergus serrator Linnaeus, 1758 & M irr, W irr & & \\
\hline Tadorna tadorna (Linnaeus, 1758) & M reg, $\mathrm{W}$ irr & VU & \\
\hline Tadorna ferruginea (Pallas, 1764) & A & & $\begin{array}{l}1 \text { ind., 16.8.2012, M. Cento, F. Fraticelli; } 1 \text { ind., 31.8.2012, S. Di Carlo; } 2 \\
\text { ind., 14.5.2014, A. Boano }\end{array}$ \\
\hline Netta rufina (Pallas, 1773) & $\begin{array}{l}\text { M irr, W irr, } \\
\text { B? }\end{array}$ & VU & Battisti \& Sorace, 2006; Battisti et al., 2020e \\
\hline Aythya ferina (Linnaeus, 1758) & $\begin{array}{l}\text { M reg, } \\
\text { W irr }\end{array}$ & VU & $\begin{array}{l}\text { Battisti et al., 2005; Battisti, 2006b; Battisti \& Sorace, 2006; Battisti et al., } \\
\text { 2006; Rizzo et al., 2006; Rizzo \& Battisti, 2007b }\end{array}$ \\
\hline
\end{tabular}




\begin{tabular}{|c|c|c|c|}
\hline Taxa & Phenology & Red List & $\begin{array}{l}\text { Notes, bibliographic references (in chronological order) and personal } \\
\text { observations (nr. of individuals, date, names of observers) }\end{array}$ \\
\hline Aythya nyroca (Güldenstädt, 1770) & $\begin{array}{l}\text { M reg, } \\
\text { W irr }\end{array}$ & EN & $\begin{array}{l}\text { Max: } 72 \text { ind. 23.3.2011, P. Gennari, C. Battisti; Battisti et al., 2004, 2005; } \\
\text { Battisti, 2006b; Battisti \& Sorace, 2006; Battisti et al., 2006; Rizzo et al., } \\
\text { 2006; Battisti et al., 2016a, 2012b }\end{array}$ \\
\hline Aythya fuligula (Linnaeus, 1758) & M irr & VU & \\
\hline Spatula querquedula (Linnaeus, 1758) & M reg & VU & $\begin{array}{l}\text { Battisti et al., 2004, 2005; Battisti, 2006b; Battisti \& Sorace, 2006; Battisti } \\
\text { et al., 2006; Rizzo et al., 2006; Lorenzetti \& Taffon, 2007; Rizzo \& Battisti, } \\
\text { 2007b, 2009; Redolfi De Zan et al., 2011; Battisti et al., 2016a }\end{array}$ \\
\hline Spatula clypeata (Linnaeus, 1758) & M reg, $\mathrm{W}$ & VU & $\begin{array}{l}\text { Battisti et al., 2004, 2005; Battisti, 2006b; Battisti \& Sorace, 2006; Battisti } \\
\text { et al., 2006; Rizzo et al., 2006; Rizzo \& Battisti, 2007b; Battisti et al., } \\
\text { 2009a; Malavasi et al., 2009a, 2009b; Rizzo \& Battisti, 2009; Battisti et al., } \\
\text { 2016a }\end{array}$ \\
\hline Mareca strepera (Linnaeus, 1758) & M reg, W irr & & Battisti, 2006b; Battisti \& Sorace, 2006; Battisti et al., 2016a \\
\hline Anas platyrhynchos Linnaeus, 1758 & $\begin{array}{c}\text { M reg, W, } \\
\text { S, B }\end{array}$ & & $\begin{array}{l}\text { Domestic form also present; Battisti et al., 2004, 2005; Battisti \& Sorace, } \\
\text { 2006; Battisti, 2006b; Battisti et al., 2006; Causarano et al., 2006; Malavasi } \\
\text { et al., 2006; Rizzo et al., 2006; Benassi et al., 2007; Lorenzetti \& Taffon, } \\
\text { 2007; Rizzo \& Battisti, 2007b; Fortunati \& Battisti, 2008; Battisti et al., } \\
\text { 2009a; Benassi \& Battisti, 2009; Causarano \& Battisti, 2009; Rizzo \& } \\
\text { Battisti, 2009; Sozio \& Battisti, 2009; Causarano et al., 2009; Malavasi } \\
\text { et al., 2009a, 2009b; Redolfi De Zan et al., 2010, 2011; Angelici et al., } \\
\text { 2012; Battisti et al., 2012a; Battisti, 2014b; Battisti et al., 2016a; Battisti \& } \\
\text { Zocchi, 2018; Battisti } \text { et al., 2020b }\end{array}$ \\
\hline Anas acuta Linnaeus, 1758 & M irr, W irr & & $\begin{array}{l}\text { Battisti et al., 2005; Battisti, 2006b; Battisti \& Sorace, 2006; Battisti et al., } \\
\text { 2006; Rizzo et al., 2006; Rizzo \& Battisti, 2007b; Battisti } \text { et al., 2016a }\end{array}$ \\
\hline Anas crecca Linnaeus, 1758 & M reg, W & EN & $\begin{array}{l}\text { Battisti et al., 2004, 2005; Battisti, 2006b; Battisti \& Sorace, 2006; Battisti } \\
\text { et al., 2006; Causarano et al., 2006; Malavasi et al., 2006; Rizzo et al., } \\
\text { 2006; Rizzo \& Battisti, 2007b; Battisti et al., 2009a; Rizzo \& Battisti, 2009; } \\
\text { Malavasi et al., 2009a, 2009b; Redolfi De Zan et al., 2010, 2011; Battisti et } \\
\text { al., 2016a }\end{array}$ \\
\hline Podiceps cristatus (Linnaeus, 1758) & $\mathrm{M}$ reg, $\mathrm{W}$ & & Battisti \& Sorace, 2006; Battisti et al., 2016a \\
\hline Podiceps nigricollis C. L. Brehm, 1831 & A & & $\begin{array}{l}3 \text { ind., 2.12.2009, S. Di Carlo; } 3 \text { ind., 17.2.2011, S. Hueting; } 1 \text { ind., } \\
\text { 8.12.2006, M. Coppola; } 2 \text { ind., 15.1.2007, G. Grillo; } 1 \text { ind., 22.12.2007, M. } \\
\text { Cento }\end{array}$ \\
\hline \multicolumn{4}{|l|}{ PHOENICOPTERIFORMES } \\
\hline \multicolumn{4}{|l|}{ Phoenicopteridae } \\
\hline Phoenicopterus roseus Pallas, 1811 & $\mathrm{M}$ irr, W irr & & Battisti, 2006b; Battisti \& Sorace, 2006; Lorenzetti \& Taffon, 2007 \\
\hline \multicolumn{4}{|l|}{ COLUMBIFORMES } \\
\hline \multicolumn{4}{|l|}{ Columbidae } \\
\hline Columba palumbus Linnaeus, 1758 & M irr & & \\
\hline Streptopelia turtur (Linnaeus, 1758) & M reg & & Battisti \& Sorace, 2006; Sorace et al., 2015 \\
\hline $\begin{array}{l}\text { Streptopelia decaocto (Frivaldszky, } \\
1838 \text { ) }\end{array}$ & $\mathrm{S}, \mathrm{B}$ & & $\begin{array}{l}\text { Battisti, 2006b; Battisti \& Sorace, 2006; Malavasi et al., 2006; Fortunati \& } \\
\text { Battisti, 2008; Battisti et al., 2009a; Malavasi et al., 2009a, 2009b; Sozio \& } \\
\text { Battisti, 2009; Battisti et al., 2016a, 2019a, 2020b }\end{array}$ \\
\hline \multicolumn{4}{|l|}{ CAPRIMULGIFORMES } \\
\hline \multicolumn{4}{|l|}{ Caprimulgidae } \\
\hline
\end{tabular}




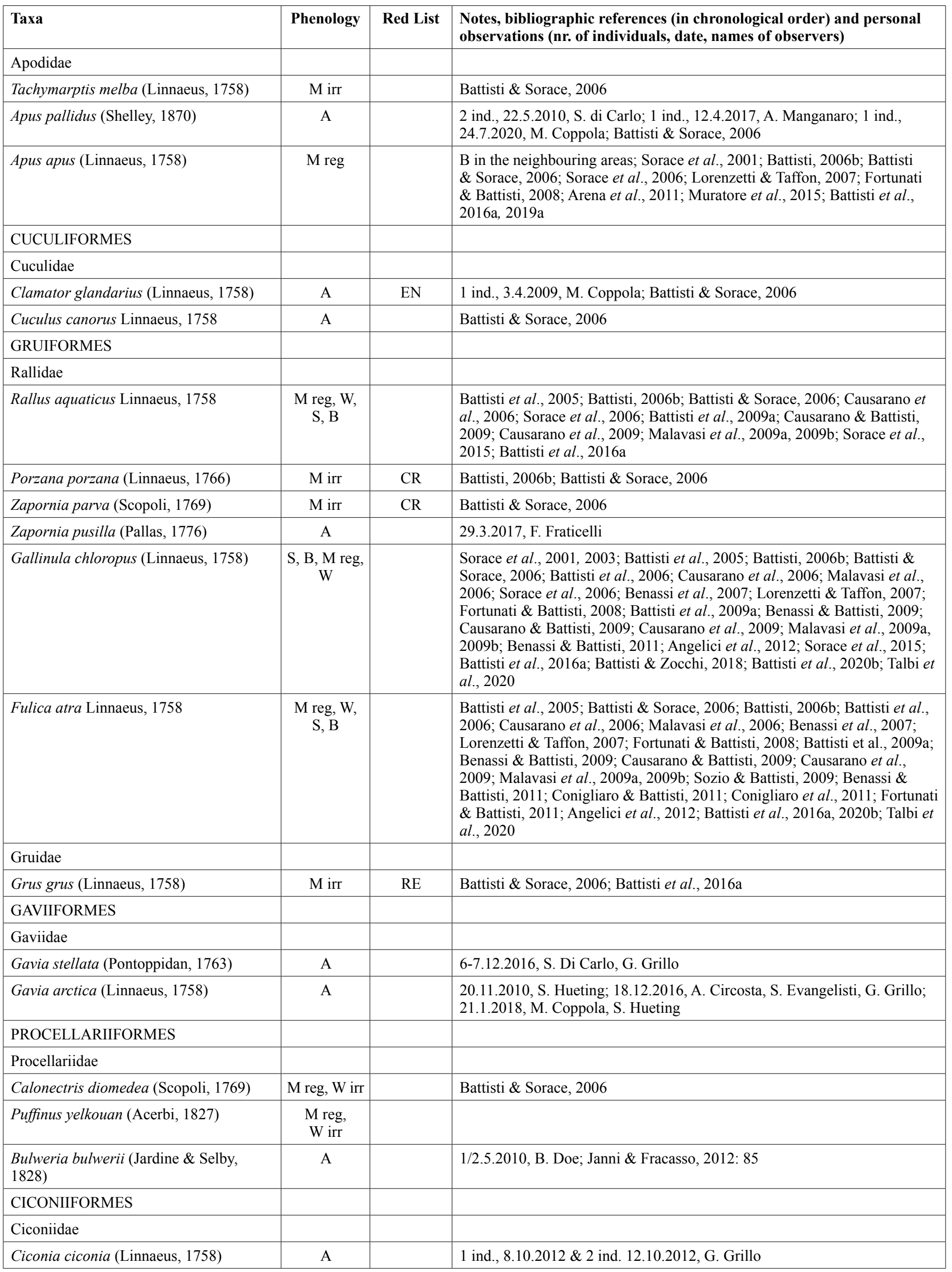




\begin{tabular}{|c|c|c|c|}
\hline Taxa & Phenology & Red List & $\begin{array}{l}\text { Notes, bibliographic references (in chronological order) and personal } \\
\text { observations (nr. of individuals, date, names of observers) }\end{array}$ \\
\hline \multicolumn{4}{|l|}{ PELECANIFORMES } \\
\hline \multicolumn{4}{|l|}{ Threskiornithidae } \\
\hline Platalea leucorodia Linnaeus, 1758 & $\begin{array}{l}\text { M reg, } \\
\text { W irr }\end{array}$ & & Battisti \& Sorace, 2006; Battisti et al., 2016a \\
\hline Plegadis falcinellus (Linnaeus, 1766) & $\begin{array}{l}\text { M reg, } \\
\text { W irr }\end{array}$ & VU & $\begin{array}{l}\text { 27.3.1933 (historical record for Campo di mare): Brunelli et al., 2001; } 39 \\
\text { ind. 13.4.1974: Bologna et al., 1974; see also Biondi et al., 1992; Battisti \& } \\
\text { Sorace, 2006; Lorenzetti \& Taffon, } 2007\end{array}$ \\
\hline \multicolumn{4}{|l|}{ Ardeidae } \\
\hline Botaurus stellaris (Linnaeus, 1758) & $\begin{array}{l}\text { M reg, W, } \\
\text { S irr }\end{array}$ & EN & $\begin{array}{l}\text { Battisti et al., 2005; Battisti, 2006b; Battisti \& Sorace, 2006; Battisti et al., } \\
\text { 2006; Rizzo et al., 2006; Battisti et al., 2016a }\end{array}$ \\
\hline Ixobrychus minutus (Linnaeus, 1766) & M reg, B & VU & $\begin{array}{l}\text { Sorace } \text { et al., 2001; Battisti et al., 2004, 2005; Battisti, 2006b; Battisti \& } \\
\text { Sorace, 2006; Battisti et al., 2006; Rizzo et al., 2006; Causarano et al., } \\
\text { 2006; Malavasi et al., 2006; Sorace } \text { et al., 2006; Benassi et al., 2007; } \\
\text { Lorenzetti \& Taffon, 2007; Rizzo \& Battisti, 2007b; Battisti et al., 2009a; } \\
\text { Benassi et al., 2009; Causarano et al., 2009; Malavasi et al., 2009a, 2009b; } \\
\text { Sozio \& Battisti, 2009; Benassi \& Battisti, 2011; Zacchei et al., 2011; } \\
\text { Sorace } \text { et al., 2015; Battisti et al., 2016a, 2020b }\end{array}$ \\
\hline Nycticorax nycticorax (Linnaeus, 1758) & M reg, B irr & & $\begin{array}{l}2 \text { breeding pairs, 2017, 2018, 2019, 2020; Battisti et al., 2005; Battisti, } \\
\text { 2006b; Battisti \& Sorace, 2006; Battisti et al., 2006; Rizzo et al., 2006; } \\
\text { Zacchei et al., 2011; Battisti et al., 2016a; Cento et al., } 2017\end{array}$ \\
\hline Ardeola ralloides (Scopoli, 1769) & M reg & & $\begin{array}{l}\text { Battisti et al., 2004, 2005; Battisti, 2006b; Battisti \& Sorace, 2006; Battisti } \\
\text { et al., 2006; Rizzo et al., 2006; Lorenzetti \& Taffon, 2007; Rizzo \& Battisti, } \\
\text { 2007b; Zacchei et al., 2011; Battisti et al., 2016a }\end{array}$ \\
\hline Bubulcus ibis (Linnaeus, 1758) & $\mathrm{B} ?, \mathrm{~W}$ & & $\begin{array}{l}\text { Roost with at least } 198 \text { ind., 10.2020; C. Battisti, M. Perchinelli; Battisti et } \\
\text { al., 2004, 2005; Battisti \& Sorace, 2006; Battisti et al., 2006; Rizzo et al., } \\
\text { 2006; Zacchei et al., 2011; Battisti et al., 2016a }\end{array}$ \\
\hline Ardea cinerea Linnaeus, 1758 & M reg, W, S & & $\begin{array}{l}\text { Battisti et al., 2004, 2005; Battisti, 2006b; Battisti \& Sorace, 2006; Battisti } \\
\text { et al., 2006; Rizzo et al., 2006; Lorenzetti \& Taffon, 2007; Rizzo \& Battisti, } \\
\text { 2007b; Battisti et al., 2009a; Redolfi De Zan et al., 2011; Zacchei et al., } \\
\text { 2011; Battisti } \text { et al., 2012a; Battisti, 2014b; Battisti et al., 2016a }\end{array}$ \\
\hline Egretta garzetta (Linnaeus, 1766) & M reg, $\mathrm{W}, \mathrm{S}$ & & $\begin{array}{l}\text { Battisti et al., 2004, 2005; Battisti, 2006b; Battisti \& Sorace, 2006; Battisti } \\
\text { et al., 2006; Rizzo et al., 2006; Lorenzetti \& Taffon, 2007; Rizzo \& Battisti, } \\
\text { 2007a, 2007b; Redolfi De Zan et al., 2011; Zacchei et al., 2011; Battisti et } \\
\text { al., 2016a }\end{array}$ \\
\hline \multicolumn{4}{|l|}{ SULIFORMES } \\
\hline \multicolumn{4}{|l|}{ Sulidae } \\
\hline Morus bassanus (Linnaeus, 1758) & M reg, W irr & & Battisti \& Sorace, 2006 \\
\hline \multicolumn{4}{|l|}{ Phalacrocoracidae } \\
\hline Phalacrocorax carbo (Linnaeus, 1758) & M reg, $\mathrm{W}$ & & $\begin{array}{l}\text { Battisti, 2006b; Battisti \& Sorace, 2006; Battisti et al., 2009a; Malavasi et } \\
\text { al., 2009a; Battisti et al., 2016a }\end{array}$ \\
\hline \multicolumn{4}{|l|}{ CHARADRIIFORMES } \\
\hline \multicolumn{4}{|l|}{ Burhinidae } \\
\hline Burhinus oedicnemus (Linnaeus, 1758) & A & & 25.4.2011, B. Doe; Battisti et al., 2015a \\
\hline \multicolumn{4}{|l|}{ Haematopodidae } \\
\hline Haematopus ostralegus Linnaeus, 1758 & M reg & VU & Battisti, 2006b; Battisti \& Sorace, 2006; Lorenzetti \& Taffon, 2007 \\
\hline \multicolumn{4}{|l|}{ Recurvirostridae } \\
\hline Recurvirostra avosetta Linnaeus, 1758 & M irr & & Battisti \& Sorace, 2006; Battisti et al., 2015a \\
\hline
\end{tabular}




\begin{tabular}{|c|c|c|c|}
\hline Taxa & Phenology & Red List & $\begin{array}{l}\text { Notes, bibliographic references (in chronological order) and personal } \\
\text { observations (nr. of individuals, date, names of observers) }\end{array}$ \\
\hline \multicolumn{4}{|l|}{ Charadriidae } \\
\hline Pluvialis apricaria (Linnaeus, 1758) & $\mathrm{M}$ irr, $\mathrm{W}$ irr & & $\begin{array}{l}\text { Max 150, 7.2.2004, R. Gildi, V. Dundee; W reg in the neighbouring areas } \\
\text { (Macchiatonda); Battisti et al., 2015a, 2016a }\end{array}$ \\
\hline Charadrius hiaticula Linnaeus, 1758 & $\begin{array}{l}\text { M reg, W } \\
\text { irr }\end{array}$ & & $\begin{array}{l}\text { Battisti \& Sorace, 2006; Battisti et al., 2009a; Malavasi et al., 2009a, } \\
\text { 2009b; Battisti et al., 2015a, 2019b }\end{array}$ \\
\hline Charadrius dubius Scopoli, 1786 & $\begin{array}{l}\text { M reg, B, } \\
\text { W irr }\end{array}$ & & $\begin{array}{l}\text { Biondi \& Pietrelli, 1999; Battisti, 2006b; Battisti \& Sorace, 2006; } \\
\text { Causarano et al., 2006; Lorenzetti \& Taffon, 2007; Fortunati \& Battisti, } \\
\text { 2008; Biondi \& Pietrelli, 2009; Causarano et al., 2009; Sozio \& Battisti, } \\
\text { 2009; Battisti et al., 2011, 2012a; Battisti, 2014b; Fraticelli, 2014; Battisti } \\
\text { et al., 2015a, 2016a, 2018a, 2020b }\end{array}$ \\
\hline Vanellus vanellus (Linnaeus, 1758) & $\mathrm{M}$ reg, $\mathrm{W}$ & & $\begin{array}{l}\text { Battisti, 2006b; Battisti \& Sorace, 2006; Battisti et al., 2009a; Malavasi et } \\
\text { al., 2009a, 2009b; Battisti et al., 2015a, 2016a }\end{array}$ \\
\hline Scolopacidae & & & Battisti \& Sorace, 2006 \\
\hline Numenius phaeopus (Linnaeus, 1758) & M reg & & $\begin{array}{l}\text { Max } 85 \text { ind. 8.4.2004, R. Gildi, V. Dundee; Battisti \& Sorace, 2006; Battisti } \\
\text { et al., 2015a, 2016a }\end{array}$ \\
\hline Numenius arquata (Linnaeus, 1758) & $\mathrm{M}$ irr, $\mathrm{W}$ irr & & Battisti \& Sorace, 2006 \\
\hline Limosa lapponica (Linnaeus, 1758) & A & & 5.4.2011, A. Circosta; 8.9.2012, M. Cento \\
\hline Limosa limosa (Linnaeus, 1758) & M irr & EN & $\begin{array}{l}\text { Also ssp. islandica C. L. Brehm, 1831; Battisti \& Sorace, 2006; Lorenzetti } \\
\text { \& Taffon, 2007; Battisti et al., 2016a }\end{array}$ \\
\hline Calidris temminckii (Leisler, 1812) & M irr & & \\
\hline Calidris alba (Pallas, 1764) & M reg, W irr & & \\
\hline Calidris alpina (Linnaeus, 1758) & M reg, W irr & & $\begin{array}{l}\text { Battisti, 2006b; Battisti \& Sorace, 2006; Battisti et al., 2015a; Sorace et al., } \\
\text { 2015; Battisti et al., 2016a }\end{array}$ \\
\hline Calidris minuta (Leisler, 1812) & M reg & & Battisti \& Sorace, 2006; Fraticelli, 2014; Battisti et al., 2015a, 2016a \\
\hline Gallinago gallinago (Linnaeus, 1758) & M reg, $\mathrm{W}$ & & $\begin{array}{l}\text { Sorace } \text { et al., 2003; Battisti, 2006b; Battisti \& Sorace, 2006; Sorace et } \\
\text { al., 2006; Lorenzetti \& Taffon, 2007; Battisti } \text { et al., 2009a; Malavasi et } \\
\text { al., 2009a, 2009b; Battisti et al., 2015a; Sorace et al., 2015; Battisti et al., } \\
\text { 2016a }\end{array}$ \\
\hline Lymnocryptes minimus (Brünnich, 1764) & M irr, W irr & & Battisti, 2006b; Battisti \& Sorace, 2006; Battisti et al., 2016a \\
\hline Actitis hypoleucos (Linnaeus, 1758) & $\begin{array}{l}\text { M reg, } S \\
\text { W irr }\end{array}$ & & $\begin{array}{l}\text { Battisti, 2006b; Battisti \& Sorace, 2006; Sorace et al., 2006; Lorenzetti \& } \\
\text { Taffon, 2007; Battisti et al., 2012a; Battisti, 2014b; Fraticelli, 2014; Battisti } \\
\text { et al., 2015a, 2016a }\end{array}$ \\
\hline Tringa ochropus Linnaeus, 1758 & M reg & & $\begin{array}{l}\text { Battisti, 2006b; Battisti \& Sorace, 2006; Lorenzetti \& Taffon, 2007; Battisti } \\
\text { et al., 2015a, 2016a }\end{array}$ \\
\hline Tringa erythropus (Pallas, 1764) & M irr & & Battisti \& Sorace, 2006; Battisti et al., 2015a \\
\hline Tringa nebularia (Gunnerus, 1767) & M reg & & Battisti \& Sorace, 2006; Battisti et al., 2015a, 2016a \\
\hline Tringa totanus (Linnaeus, 1758) & M reg & & Battisti \& Sorace, 2006; Battisti et al., 2015a, 2016a \\
\hline Tringa glareola Linnaeus, 1758 & M reg & & $\begin{array}{l}\text { Battisti \& Sorace, 2006; Sorace et al., 2006; Lorenzetti \& Taffon, 2007; } \\
\text { Battisti et al., 2015a, 2016a }\end{array}$ \\
\hline
\end{tabular}




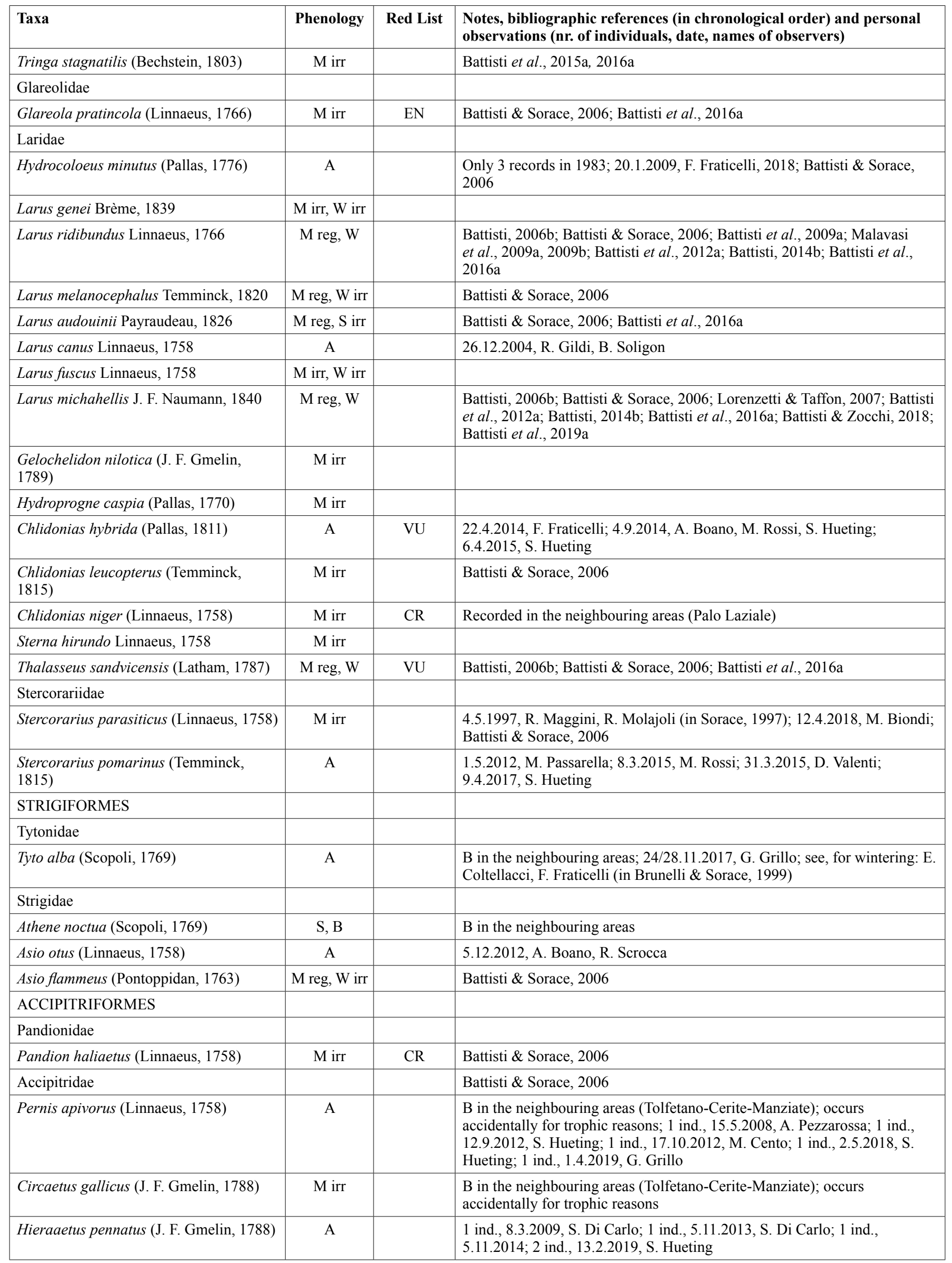




\begin{tabular}{|c|c|c|c|}
\hline Taxa & Phenology & Red List & $\begin{array}{l}\text { Notes, bibliographic references (in chronological order) and personal } \\
\text { observations (nr. of individuals, date, names of observers) }\end{array}$ \\
\hline Circus aeruginosus (Linnaeus, 1758) & M reg, $\mathrm{W}$ & VU & $\begin{array}{l}\text { Battisti, 2006b; Battisti \& Sorace, 2006; Battisti, 2007; Lorenzetti \& Taffon, } \\
\text { 2007; Battisti et al., 2009a; Malavasi et al., 2009a, 2009b; Angelici et al., } \\
\text { 2012; Battisti et al., 2016a }\end{array}$ \\
\hline Circus cyaneus (Linnaeus, 1766) & $\mathrm{M}$ irr, W irr & & Battisti \& Sorace, 2006; 11.2007, R. Lippolis \\
\hline Circus pygargus (Linnaeus, 1758) & A & VU & $\begin{array}{l}1 \text { ind., 10.4.2003, E. Savo; } 1 \text { ind., 7.11.2009, S. di Carlo; } 1 \text { ind., 4.5.2011, } \\
\text { A. Circosta; Battisti \& Sorace, } 2006\end{array}$ \\
\hline Milvus milvus (Linnaeus, 1758) & $\begin{array}{l}\text { M reg, } \\
\text { W irr }\end{array}$ & VU & $\begin{array}{l}\text { B in the neighbouring areas (Tolfetano-Cerite-Manziate); occurs } \\
\text { accidentally for trophic reasons; Battisti \& Sorace, } 2006\end{array}$ \\
\hline Milvus migrans (Boddaert, 1783) & $\mathrm{M}$ reg & & $\begin{array}{l}\mathrm{B} \text { in the neighbouring areas (Tolfetano-Cerite-Manziate); occurs } \\
\text { accidentally for trophic reasons }\end{array}$ \\
\hline Upupa epops Linnaeus, 1758 & M reg & & Battisti \& Sorace, 2006; Battisti et al., 2016a \\
\hline \multicolumn{4}{|l|}{ CORACIIFORMES } \\
\hline \multicolumn{4}{|l|}{ Meropidae } \\
\hline Merops apiaster Linnaeus, 1758 & M reg & & Battisti, 2006b; Battisti \& Sorace, 2006 \\
\hline \multicolumn{4}{|l|}{ Alcedinidae } \\
\hline Alcedo atthis Linnaeus, 1758 & $\begin{array}{l}\text { M reg, W, } \\
\quad \mathrm{S}, \mathrm{B} \text { ? }\end{array}$ & & $\begin{array}{l}\text { Sorace et al., 2001, 2003; Battisti, 2006b; Battisti \& Sorace, 2006; Sorace } \\
\text { et al., 2006; Benassi et al., 2007; Battisti et al., 2009a; Malavasi et al., } \\
\text { 2009a, 2009b; Sorace et al., 2010, 2015; Battisti et al., 2016a }\end{array}$ \\
\hline \multicolumn{4}{|l|}{ PICIFORMES } \\
\hline Falco eleonorae Gené, 1839 & A & VU & 2 ind., 16.5.2008, M. Biondi; 1 ind., 2.6.2009, B. Doe \\
\hline Falco columbarius Linnaeus, 1758 & $\mathrm{M}$ irr, $\mathrm{W}$ irr & & Sorace et al., 2003; Battisti \& Sorace, 2006; Sorace et al., 2006, 2015 \\
\hline Falco subbuteo Linnaeus, 1758 & M irr & & Battisti, 2006b; Battisti \& Sorace, 2006 \\
\hline Falco cherrug J. E. Gray, 1834 & A & & Battisti \& Sorace, 2006; 17.3.2017: R. Scrocca (in Condello, 2018) \\
\hline Falco biarmicus Temminck, 1825 & A & & 1 ind., 9.3.2012, R. Scrocca \\
\hline Falco peregrinus Tunstall, 1771 & $\begin{array}{l}\text { M reg, } \\
\text { W irr }\end{array}$ & & $\begin{array}{l}\text { B in the neighbouring areas (Tolfetano-Cerite-Manziate); occurs } \\
\text { accidentally for trophic reasons; Battisti \& Sorace, } 2006\end{array}$ \\
\hline \multicolumn{4}{|l|}{ PASSERIFORMES } \\
\hline \multicolumn{4}{|l|}{ Laniidae } \\
\hline Lanius collurio Linnaeus, 1758 & $\mathrm{M}$ irr, B? & VU & Battisti \& Sorace, 2006; Sorace et al., 2006; Battisti et al., 2020b \\
\hline Lanius minor J. F. Gmelin, 1788 & A & EN & 3.7.2012, M. Cento \\
\hline Lanius excubitor Linnaeus, 1758 & A & EN & 7.12.2017, G. Grillo \\
\hline Lanius senator Linnaeus, 1758 & M irr & & $\begin{array}{l}\text { Both ssp. badius Hartlaub, } 1854 \text { and ssp. senator Linnaeus, 1758, observed } \\
\text { in the neighbouring areas (Palo e Macchiatonda); Battisti \& Sorace, 2006; } \\
\text { Battisti et al., 2016a }\end{array}$ \\
\hline \multicolumn{4}{|l|}{ Corvidae } \\
\hline Pica pica (Linnaeus, 1758) & $\mathrm{S}, \mathrm{B}$ & & $\begin{array}{l}\text { Battisti, 2006b; Battisti \& Sorace, 2006; Sorace } \text { et al., 2006; Malavasi et al., } \\
\text { 2006; Battisti \& Sorace, 2006; Lorenzetti \& Taffon, 2007; Fortunati \& Battisti, } \\
\text { 2008; Battisti et al., 2009a; Malavasi et al., 2009a, 2009b; Sozio \& Battisti, } \\
\text { 2009; Battisti et al., 2012a; Battisti, 2014b; Battisti et al., 2016a, 2019a, 2020b }\end{array}$ \\
\hline
\end{tabular}




\begin{tabular}{|c|c|c|c|}
\hline Taxa & Phenology & Red List & $\begin{array}{l}\text { Notes, bibliographic references (in chronological order) and personal } \\
\text { observations (nr. of individuals, date, names of observers) }\end{array}$ \\
\hline Corvus monedula Linnaeus, 1758 & $\mathrm{~S}$ & & $\begin{array}{l}\text { B in the neighbouring areas; Battisti, 2006b; Battisti \& Sorace, 2006; } \\
\text { Battisti et al., 2016a }\end{array}$ \\
\hline \multicolumn{4}{|l|}{ Paridae } \\
\hline Cyanistes caeruleus (Linnaeus, 1758) & W, S, B & & $\begin{array}{l}\text { Sorace et al., 2001, 2003; Battisti \& Sorace, 2006; Sorace et al., 2006, } \\
\text { 2015; Battisti et al., 2016a }\end{array}$ \\
\hline Parus major Linnaeus, 1758 & S, B & & $\begin{array}{l}\text { Sorace et al., 2001; Battisti, 2006b; Battisti \& Sorace, 2006; Sorace et al., } \\
\text { 2006; Fortunati \& Battisti, 2008; Sozio \& Battisti, 2009; Sorace } \text { et al., } \\
\text { 2015; Battisti et al., 2016a, 2019a, 2020b }\end{array}$ \\
\hline \multicolumn{4}{|l|}{ Alaudidae } \\
\hline $\begin{array}{l}\text { Calandrella brachydactyla (Leisler, } \\
\text { 1814) }\end{array}$ & A & & $\begin{array}{l}\text { B in the neighbouring areas; } 1 \text { ind., 12.4.2013, F. Fraticelli; } 1 \text { ind., } \\
\text { 23.4.2015, S. Hueting; Battisti \& Sorace, 2006; Fortunati \& Battisti, } 2008\end{array}$ \\
\hline Lullula arborea (Linnaeus, 1758) & M irr & & \\
\hline Alauda arvensis Linnaeus, 1758 & $\begin{array}{l}\text { M reg, W, } \\
\text { S, B }\end{array}$ & & $\begin{array}{l}\text { Battisti, 2006b; Battisti \& Sorace, 2006; Malavasi et al., 2006; Battisti \& } \\
\text { Sorace, 2006; Lorenzetti \& Taffon, 2007; Fortunati \& Battisti, 2008; Battisti } \\
\text { et al., 2009a; Malavasi et al., 2009a, 2009b; Battisti } \text { et al., 2016a, 2020b }\end{array}$ \\
\hline Galerida cristata (Linnaeus, 1758) & S, B & & $\begin{array}{l}\text { Battisti, 2006b; Battisti \& Sorace, 2006; Causarano et al., 2006; Malavasi et } \\
\text { al., 2006; Sorace } \text { et al., 2006; Battisti \& Sorace, 2006; Lorenzetti \& Taffon, } \\
\text { 2007; Fortunati \& Battisti, 2008; Battisti et al., 2009a; Causarano et al., } \\
\text { 2009; Malavasi et al., 2009a, 2009b; Sozio \& Battisti, 2009; Battisti et al., } \\
\text { 2012a; Battisti, 2014b; Battisti et al., 2016a, 2019a, 2020b }\end{array}$ \\
\hline \multicolumn{4}{|l|}{ Panuridae } \\
\hline \multicolumn{4}{|l|}{ Acrocephalidae } \\
\hline Hippolais polyglotta (Vieillot, 1817) & M irr & & 18.5.2011, S. Hueting \\
\hline Hippolais icterina (Vieillot, 1817) & M irr & & 26.4.2011, S. Hueting \\
\hline $\begin{array}{l}\text { Acrocephalus melanopogon (Temminck, } \\
1823 \text { ) }\end{array}$ & M reg, W & EN & $\begin{array}{l}\text { Sorace } \text { et al., 2001, 2003; Battisti, 2006b; Battisti \& Sorace, 2006; Sorace } \\
\text { et al., 2006, 2010, 2015; Battisti et al., 2016a }\end{array}$ \\
\hline $\begin{array}{l}\text { Acrocephalus schoenobaenus (Linnaeus, } \\
1758 \text { ) }\end{array}$ & M irr & EN & Sorace et al., 2001, 2006, 2015; Battisti et al., 2016a \\
\hline $\begin{array}{l}\text { Acrocephalus scirpaceus (Hermann, } \\
\text { 1804) }\end{array}$ & M reg, $\mathrm{B}$ & & $\begin{array}{l}\text { Sorace et al., 2001, 2003; Battisti, 2006b; Battisti \& Sorace, 2006; } \\
\text { Causarano et al., 2006; Malavasi et al., 2006; Sorace et al., 2006; Benassi } \\
\text { et al., 2007; Battisti \& Sorace, 2006; Lorenzetti \& Taffon, 2007; Fortunati } \\
\text { \& Battisti, 2008; Battisti et al., 2009a; Benassi et al., 2009; Causarano } \text { et } \\
\text { al., 2009; Malavasi et al., 2009a, 2009b; Sozio \& Battisti, 2009; Sorace } \\
\text { et al., 2010; Benassi \& Battisti, 2011; Ranchelli et al., 2011; Sozio et al., } \\
\text { 2013; Sorace } \text { et al., 2015; Battisti et al., 2016a, 2019a, 2020a, 2020b }\end{array}$ \\
\hline Acrocephalus agricola (Jerdon, 1845) & A & & $\begin{array}{l}\text { 29.3.2003, S. Laurenti (Torre Flavia ringing station); see Laurenti, 2002; } \\
\text { Battisti \& Sorace, 2006; Sorace et al., 2006; Brunelli \& Fraticelli, 2010 }\end{array}$ \\
\hline $\begin{array}{l}\text { Acrocephalus arundinaceus (Linnaeus, } \\
\text { 1758) }\end{array}$ & M reg, B & & $\begin{array}{l}\text { Sorace et al., 2001, 2003; Battisti, 2006b; Battisti \& Sorace, 2006; Causarano } \\
\text { et al., 2006; Sorace et al., 2006; Benassi et al., 2007; Battisti \& Sorace, 2006; } \\
\text { Lorenzetti \& Taffon, 2007; Battisti et al., 2009a; Benassi et al., 2009; Causarano } \\
\text { et al., 2009; Sorace } \text { et al., 2010, 2015; Battisti et al., 2016a, 2020a, 2020b }\end{array}$ \\
\hline
\end{tabular}




\begin{tabular}{|c|c|c|c|}
\hline Taxa & Phenology & Red List & $\begin{array}{l}\text { Notes, bibliographic references (in chronological order) and personal } \\
\text { observations (nr. of individuals, date, names of observers) }\end{array}$ \\
\hline \multicolumn{4}{|l|}{ Locustellidae } \\
\hline Locustella luscinioides (Savi, 1824) & M irr & EN & $\begin{array}{l}\text { Battisti \& Sorace, 2006; Sorace et al., 2006; Torre Flavia ringing station, } \\
\text { 2011: Sorace et al., 2015; Battisti et al., 2016a }\end{array}$ \\
\hline \multicolumn{4}{|l|}{ Hirundinidae } \\
\hline Delichon urbicum (Linnaeus, 1758) & $\begin{array}{l}\text { M reg, } \\
\text { W irr }\end{array}$ & & $\begin{array}{l}\text { B in the neighbouring areas; Sorace } \text { et al., 2001; Battisti, 2006b; Battisti } \\
\text { \& Sorace, 2006; Sorace } \text { et al., 2006; Battisti \& Sorace, 2006; Lorenzetti \& } \\
\text { Taffon, 2007; Fortunati \& Battisti, 2008; Arena et al., 2011; Muratore } \text { et al., } \\
\text { 2015; Battisti et al., 2016a, 2019a }\end{array}$ \\
\hline Cecropis daurica (Laxmann, 1769) & M irr & VU & Battisti, 2006b; Battisti \& Sorace, 2006; Battisti et al., 2016a \\
\hline Hirundo rustica Linnaeus, 1758 & $\begin{array}{l}\text { M reg, B, } \\
\text { W irr }\end{array}$ & & $\begin{array}{l}\text { Sorace et al., 2001; Battisti, 2006b; Monti, 2006; Battisti \& Sorace, 2006; } \\
\text { Sorace et al., 2006; Battisti \& Sorace, 2006; Lorenzetti \& Taffon, 2007; } \\
\text { Fortunati \& Battisti, 2008; Arena et al., 2011; Muratore et al., 2015; Sorace } \\
\text { et al., 2015; Battisti et al., 2016a, 2019a }\end{array}$ \\
\hline Phylloscopus sibilatrix (Bechstein, 1793) & M irr & & $\begin{array}{l}\text { Sorace et al., 2001; Battisti \& Sorace, 2006; Sorace et al., 2006; Battisti et } \\
\text { al., } 2016 \mathrm{a}\end{array}$ \\
\hline Phylloscopus trochilus (Linnaeus, 1758) & M irr & & $\begin{array}{l}\text { Sorace et al., 2001; Battisti \& Sorace, 2006; Sorace et al., 2006, 2015; } \\
\text { Battisti et al., 2016a }\end{array}$ \\
\hline Phylloscopus collybita (Vieillot, 1817) & M reg, W & & $\begin{array}{l}\text { ssp. collybita Vieillot, } 1817 \text { and (probably) ssp. abietinus; Sorace } \text { et al., } \\
\text { 2001, 2003; Battisti, 2006b; Battisti \& Sorace, 2006; Sorace et al., 2006; } \\
\text { Malavasi et al., 2009a, 2009b; Sorace et al., 2010; Battisti et al., 2012a; } \\
\text { Battisti, 2014b; Sorace et al., 2015; Battisti et al., 2016a; ssp. abietinus: F. } \\
\text { Fraticelli, 16.2.2009 }\end{array}$ \\
\hline Phylloscopus tristis Blyth, 1843 & M reg, W & & 13 and 20.1.2001, F. Fraticelli (in Brunelli \& Sorace, 2001) \\
\hline \multicolumn{4}{|l|}{ Scotocercidae } \\
\hline \multicolumn{4}{|l|}{ Aegithalidae } \\
\hline Aegithalos caudatus (Linnaeus, 1758) & $\mathrm{S}, \mathrm{B}$ ? & & Battisti \& Sorace, 2006; B in the neighbouring areas \\
\hline \multicolumn{4}{|l|}{ Sylviidae } \\
\hline Sylvia atricapilla (Linnaeus, 1758) & $\mathrm{S}, \mathrm{B}, \mathrm{M}$ reg & & $\begin{array}{l}\text { Sorace et al., 2001, 2003; Battisti \& Sorace, 2006; Sorace et al., 2006, } \\
\text { 2010, 2015; Battisti et al., 2016a }\end{array}$ \\
\hline Sylvia borin (Boddaert, 1783) & $\mathrm{M}$ irr & VU & Sorace et al., 2001; Battisti \& Sorace, 2006; Sorace et al., 2006, 2015 \\
\hline Sylvia curruca (Linnaeus, 1758) & A & & Torre Flavia Ringing station (Autumn 2008); Sorace et al., 2015 \\
\hline $\begin{array}{l}\text { Sylvia melanocephala (J. F. Gmelin, } \\
1789 \text { ) }\end{array}$ & S, B & & $\begin{array}{l}\text { Sorace et al., 2001, 2003; Battisti, 2006b; Battisti \& Sorace, 2006; Malavasi } \\
\text { et al., 2006; Sorace } \text { et al., 2006; Fortunati \& Battisti, 2008; Battisti et al., } \\
\text { 2009a; Malavasi et al., 2009a, 2009b; Sorace et al., 2010, 2015; Battisti } \text { et } \\
\text { al., 2016a, 2019a, 2020b }\end{array}$ \\
\hline Sylvia cantillans (Pallas, 1764) & M reg, B? & & $\begin{array}{l}\text { ssp. cantillans, Pallas, } 1764 \text { (B?); ssp. albistriata, C. L. Brehm, } 1855 \\
\text { (Mreg); Sorace } \text { et al., 2001; Battisti \& Sorace, 2006; Sorace et al., 2006; } \\
\text { Battisti \& Sorace, 2006; Lorenzetti \& Taffon, 2007; Battisti et al., 2016a }\end{array}$ \\
\hline Sylvia subalpina Temminck, 1820 & A & & 27.4.2017, R. Scrocca \\
\hline Sylvia communis Latham, 1787 & M irr & & $\begin{array}{l}\text { Sorace et al., 2001; Battisti \& Sorace, 2006; Sorace et al., 2006; Battisti et } \\
\text { al., 2016a }\end{array}$ \\
\hline Sylvia undata (Boddaert, 1783) & A & & Torre Flavia Ringing station (Autumn 2007); Sorace et al., 2015 \\
\hline \multicolumn{4}{|l|}{ Certhiidae } \\
\hline Certhia brachydactyla C. L. Brehm, 1820 & A & & 8/20.10.2010, M. Cento, S. Hueting; Battisti et al., 2016a \\
\hline \multicolumn{4}{|l|}{ Troglodytidae } \\
\hline
\end{tabular}




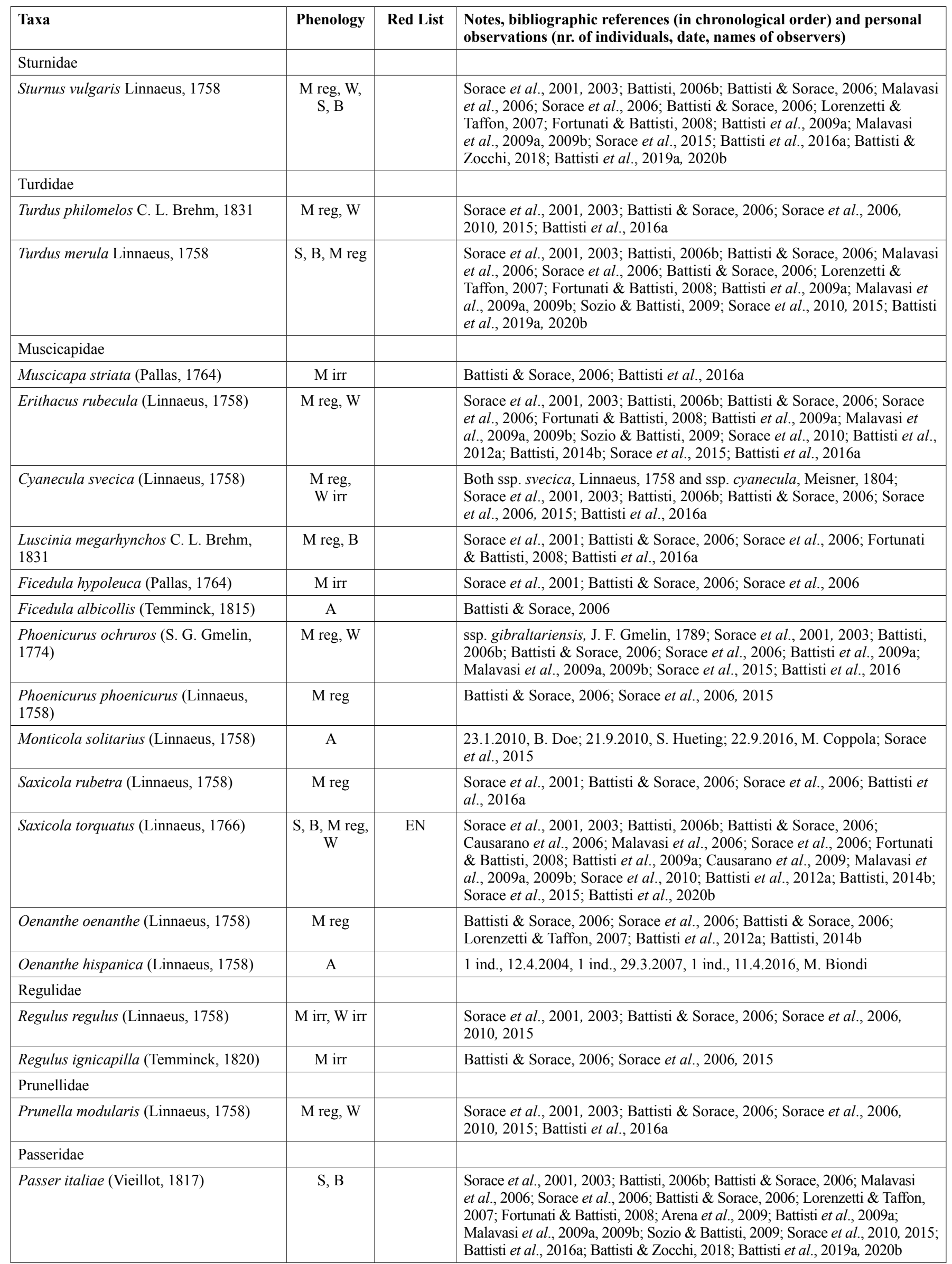




\begin{tabular}{|c|c|c|c|}
\hline Taxa & Phenology & Red List & $\begin{array}{l}\text { Notes, bibliographic references (in chronological order) and personal } \\
\text { observations (nr. of individuals, date, names of observers) }\end{array}$ \\
\hline Passer montanus (Linnaeus, 1758) & $\mathrm{S}, \mathrm{B}, \mathrm{W}$ & & $\begin{array}{l}\text { Sorace et al., 2001, 2003; Battisti, 2006b; Battisti \& Sorace, 2006; Malavasi } \\
\text { et al., 2006; Sorace et al., 2006; Fortunati \& Battisti, 2008; Battisti et al., } \\
\text { 2009a; Malavasi et al., 2009a, 2009b; Sorace et al., 2015; Battisti et al., } \\
\text { 2016a, 2019a, 2020b }\end{array}$ \\
\hline \multicolumn{4}{|l|}{ Motacillidae } \\
\hline Anthus pratensis (Linnaeus, 1758) & M reg, W & & $\begin{array}{l}\text { Fraticelli, 2014; Battisti, 2006b; Battisti \& Sorace, 2006; Sorace et al., } \\
\text { 2006; Battisti et al., 2009a; Malavasi et al., 2009a, 2009b; Battisti et al., } \\
\text { 2012a; Battisti, 2014b; Sorace } \text { et al., 2015; Battisti et al., 2016a }\end{array}$ \\
\hline Anthus spinoletta (Linnaeus, 1758) & M reg, $\mathrm{W}$ & & $\begin{array}{l}\text { Sorace et al., 2001, 2003; R. Lippolis, 11.2007; Battisti \& Sorace, 2006; } \\
\text { Sorace } \text { et al., 2006, 2010, } 2015\end{array}$ \\
\hline Anthus campestris (Linnaeus, 1758) & A & VU & 9.12.2008, S. Rempicci; Sighele \& Janni, 2010: 87 \\
\hline Motacilla flava Linnaeus, 1758 & M reg & & $\begin{array}{l}\text { Subspecies: flava Linnaeus, 1758, thunbergi Billberg, 1828, feldegg } \\
\text { Michahelles, 1830, cinereocapilla Savi, 1831, beema, Sykes, 1832; Sorace } \\
\text { et al., 2001, 2003; Battisti, 2006b; Battisti \& Sorace, 2006; Sorace et al., } \\
\text { 2006; Battisti et al., 2012a; Battisti, 2014b; Sorace et al., 2015; Battisti et } \\
\text { al., 2016a; 26.3.2017, S. Hueting }\end{array}$ \\
\hline Motacilla cinerea Tunstall, 1771 & $\begin{array}{l}\text { M reg, } \\
\text { W irr }\end{array}$ & & $\begin{array}{l}\text { Sorace et al., 2001, 2003; Battisti \& Sorace, 2006; Sorace et al., 2006, } \\
\text { 2015; Battisti et al., 2016a }\end{array}$ \\
\hline Motacilla citreola Pallas, 1776 & A & & $\begin{array}{l}\text { 22.4.2015, A Manganaro; 1.5.2016, F. Fraticelli, M. Grylle, S. Hueting; 20- } \\
\text { 25.10.2018, S. Patrizi }\end{array}$ \\
\hline Motacilla alba Linnaeus, 1758 & $\begin{array}{l}\mathrm{S}, \mathrm{B}, \mathrm{M} \text { reg, } \\
\mathrm{W}\end{array}$ & & $\begin{array}{l}\text { Also ssp. yarrellii Gould, 1837; Sorace et al., 2001, 2003; Battisti, 2006b; } \\
\text { Battisti \& Sorace, 2006; Causarano et al., 2006; Malavasi et al., 2006; } \\
\text { Sorace et al., 2006; Battisti \& Sorace, 2006; Lorenzetti \& Taffon, 2007; } \\
\text { Fortunati \& Battisti, 2008; Battisti et al., 2009a; Causarano et al., 2009; } \\
\text { Malavasi et al., 2009a, 2009b; Sozio \& Battisti, 2009; Sorace } \text { et al., 2010; } \\
\text { Battisti et al., 2012a; Battisti, 2014b; Fraticelli, 2014; Sorace } \text { et al., 2015; } \\
\text { Battisti et al., 2016a; Battisti et al., 2019a, 2020b; for ssp. yarrellii: Winter } \\
\text { 1995/1996, Fraticelli, 1997; 16.12.2001, F. Fraticelli; 27.4.2011, F. Mantero } \\
\text { (Torre Flavia ringing station) }\end{array}$ \\
\hline \multicolumn{4}{|l|}{ Fringillidae } \\
\hline Fringilla coelebs Linnaeus, 1758 & M reg, $\mathrm{W}$ & & $\begin{array}{l}\text { Sorace et al., 2001, 2003; Battisti, 2006b; Battisti \& Sorace, 2006; Sorace } \\
\text { et al., 2006; Battisti et al., 2012a; Battisti, 2014b; Sorace } \text { et al., 2015; } \\
\text { Battisti et al., } 2016\end{array}$ \\
\hline $\begin{array}{l}\text { Coccothraustes coccothraustes } \\
\text { (Linnaeus, 1758) }\end{array}$ & A & & $\begin{array}{l}1 \text { ind., 8.11.2014, M. Coppola; } 1 \text { ind., 18.1.2015, M. Coppola; } 1 \text { ind., } \\
\text { 14.1.2018, M. Cento; other data available for the neighbouring areas: } \\
\text { (Ladispoli) }\end{array}$ \\
\hline Chloris chloris (Linnaeus, 1758) & S, B, M reg & & $\begin{array}{l}\text { Sorace et al., 2001, 2003; Battisti, 2006b; Battisti \& Sorace, 2006; } \\
\text { Causarano et al., 2006; Malavasi et al., 2006; Sorace et al., 2006; Battisti } \\
\text { \& Sorace, 2006; Lorenzetti \& Taffon, 2007; Fortunati \& Battisti, 2008; } \\
\text { Battisti et al., 2009a; Causarano et al., 2009; Malavasi et al., 2009a, 2009b; } \\
\text { Sozio \& Battisti, 2009; Sorace et al., 2010; Battisti et al., 2012a; Battisti, } \\
\text { 2014b; Sorace et al., 2015; Battisti et al., 2016a, 2019a, 2020b }\end{array}$ \\
\hline Linaria cannabina (Linnaeus, 1758) & M reg, $\mathrm{W}$ irr & & Battisti et al., 2012a; Battisti, 2014b \\
\hline Carduelis carduelis (Linnaeus, 1758) & S, B, M reg & & $\begin{array}{l}\text { Sorace et al., 2001, 2003; Battisti, 2006b; Battisti \& Sorace, 2006; } \\
\text { Causarano et al., 2006; Malavasi et al., 2006; Sorace et al., 2006; Battisti } \\
\text { \& Sorace, 2006; Lorenzetti \& Taffon, 2007; Fortunati \& Battisti, 2008; } \\
\text { Battisti et al., 2009a; Causarano et al., 2009; Malavasi et al., 2009a, 2009b; } \\
\text { Sozio \& Battisti, 2009; Sorace } \text { et al., 2015; Battisti et al., 2016a, 2019a, } \\
\text { 2020b }\end{array}$ \\
\hline Serinus serinus (Linnaeus, 1766) & $\begin{array}{l}\mathrm{S}, \mathrm{B}, \mathrm{M} \text { reg, } \\
\mathrm{W}\end{array}$ & & $\begin{array}{l}\text { Battisti, 2006b; Battisti \& Sorace, 2006; Causarano et al., 2006; Sorace et } \\
\text { al., 2006; Fortunati \& Battisti, 2008; Battisti et al., 2009a; Causarano et al., } \\
\text { 2009; Malavasi et al., 2009a, 2009b; Sozio \& Battisti, 2009; Malavasi et } \\
\text { al., 2006; Battisti et al., 2016a, 2019a, 2020b }\end{array}$ \\
\hline
\end{tabular}




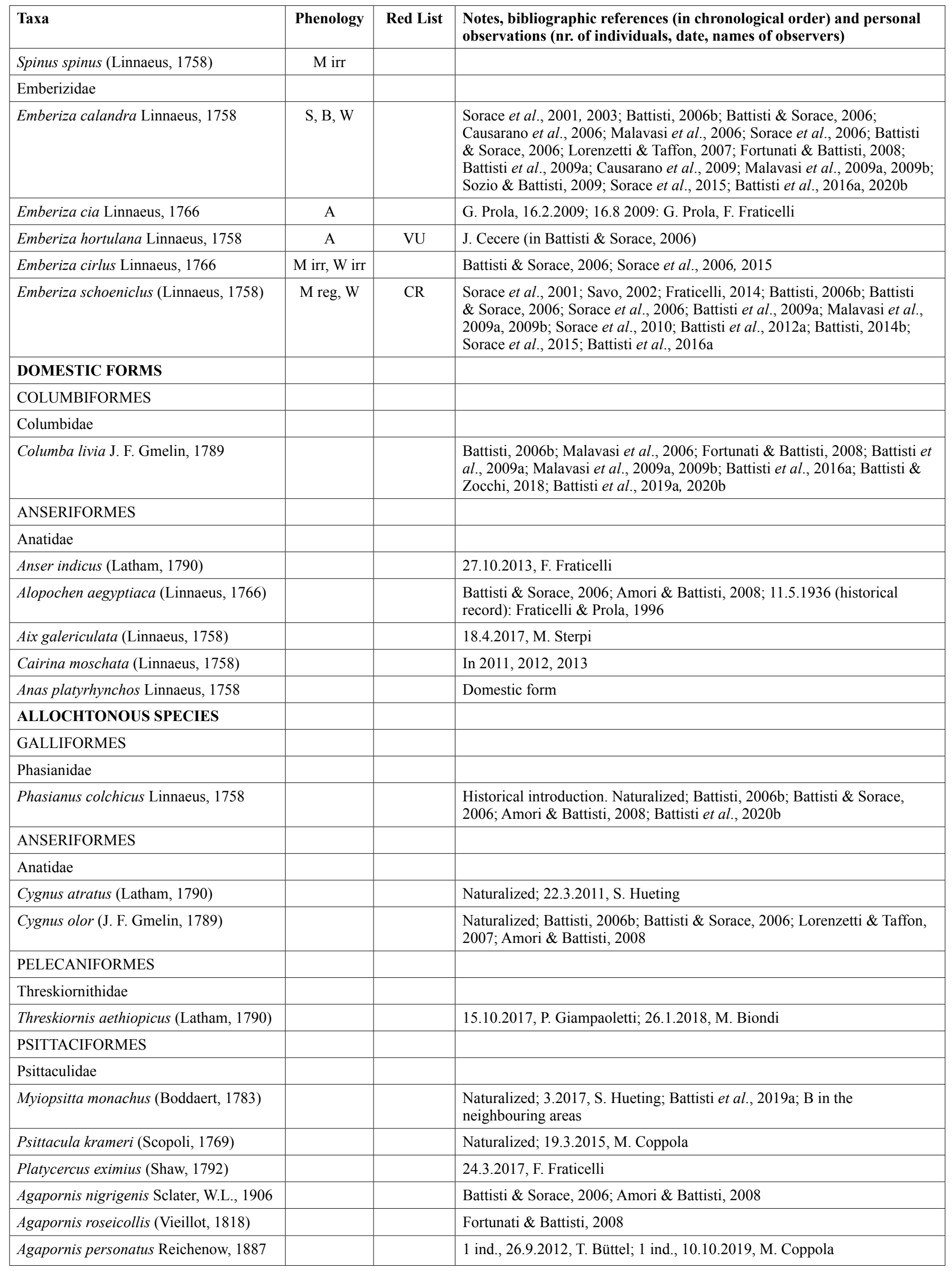




\begin{tabular}{|c|c|c|c|}
\hline Taxa & Phenology & Red List & $\begin{array}{l}\text { Notes, bibliographic references (in chronological order) and personal } \\
\text { observations (nr. of individuals, date, names of observers) }\end{array}$ \\
\hline \multicolumn{4}{|l|}{ PASSERIFORMES } \\
\hline \multicolumn{4}{|l|}{ Sturnidae } \\
\hline Acridotheres tristis (Linnaeus, 1766) & & & Battisti \& Sorace, 2006; Amori \& Battisti, 2008 \\
\hline \multicolumn{4}{|l|}{ Ploceidae } \\
\hline Quelea cardinalis (Hartlaub, 1880) & & & Battisti \& Sorace, 2006; Amori \& Battisti, 2008 \\
\hline Euplectes franciscanus (Isert, 1789) & & & Battisti \& Sorace, 2006; Amori \& Battisti, 2008 \\
\hline
\end{tabular}

\section{Mammalia}

We found records for 20 species of mammals: 18 native - one Erinaceomorpha, three Soricomorpha, five Rodentia (including three Muridae as palaeo-allochtonous species), five Chiroptera, three Carnivora and one Artiodactyla) and two non native rodents: Sciurus carolinensis (Gmelin, 1788), one individual escaped from captivity, and Myocastor coypus (Molina, 1782), as naturalized species (Tab. 5). The presence of Canis lupus Linnaeus, 1758 has been confirmed in the adjacent areas ( 2 adults and 5 juv. near Fosso Turbino - Furbara and Macchiatonda reserve, about $3 \mathrm{~km}$ to the north; S. Donfrancesco, S. Muratore, pers. comm.; July 2020). Even though in March 2020, 10 sheep were predated in the Palude di Torre Flavia by a canid, it was impossible to clearly assign the predation to a wolf: likely, the event is ascribable to a domestic dog or a wolf-dog hybrid (S. Donfrancesco, S. Esigibili, pers. comm.). For the above given reason, this species has not been included in the checklist.

The bat Miniopterus schreibersii (Kuhl, 1817) is the only species assigned to a conservation concern category (Vulnerable). Further research is necessary to improve the knowledge on the presence of some less obvious and, therefore, neglected taxa (e.g., Carnivora Mustelidae, Chiroptera).

Tab. 5 - Check list of mammals (Mammalia) occurring in the Torre Flavia wetland. Taxonomic treatment after Amori et al. (2008); Boitani et al. (2003); Lanza (2012). Among the allochthonous species, only those recently introduced are reported (therefore all murids are excluded as paleo-allocthonous species). Inclusion in global or Italian IUCN Red Lists is also reported (Rondinini et al., 2013; VU: Vulnerable; LC: Least Concern; NA: Not Applicable). / Check list dei mammiferi (Mammalia) presenti nella Palude di Torre Flavia. Trattamento tassonomico secondo Amori et al. (2008); Boitani et al. (2003); Lanza (2012). Tra le specie alloctone, solo quelle introdotte di recente sono riportate (quindi tutti i muridi sono esclusi in quanto specie paleo-alloctone). Viene riportata l'inclusione nelle Liste Rosse IUCN globale o italiana (Rondinini et al., 2013: VU = Vulnerabile; LC = Minor Preoccupazione; NA = Non Applicabile).

\begin{tabular}{|l|l|l|}
\hline Taxa & Red List & $\begin{array}{l}\text { Notes, bibliographic references (in chronological order) and personal } \\
\text { observations (nr. of individuals, date, names of observers) }\end{array}$ \\
\hline MAMMALIA & & \\
\hline Erinaceomorpha & & \\
\hline Erinaceidae & & Amori et al., 2006 \\
\hline Erinaceus europaeus Linnaeus, 1758 & & \\
\hline Soricomorpha & & Amori et al., 2006 \\
\hline Talpidae & & \\
\hline Talpa romana Thomas, 1902 & Amori et al., 2006 \\
\hline Soricidae & Amori et al., 2006 \\
\hline Suncus etruscus (Savi, 1822) & \\
\hline Crocidura suaveolens (Pallas, 1811) & & \\
\hline Rodentia & & Amori et al., 2006 \\
\hline Cricetidae & & \\
\hline Microtus savii (de Sélys-Longchamps, 1838) & Amori et al., 2006; Amori \& Battisti, 2008 \\
\hline Muridae & Amori et al., 2006; Amori \& Battisti, 2008; Angelici et al., 2012 \\
\hline Mus musculus Linnaeus, 1758 & &
\end{tabular}




\begin{tabular}{|c|c|c|}
\hline Taxa & Red List & $\begin{array}{l}\text { Notes, bibliographic references (in chronological order) and personal } \\
\text { observations (nr. of individuals, date, names of observers) }\end{array}$ \\
\hline Rattus rattus (Linnaeus, 1758) & & Amori et al., 2006 \\
\hline \multicolumn{3}{|l|}{ Hystricidae } \\
\hline Hystrix cristata Linnaeus, 1758 & & Amori et al., 2006 \\
\hline \multicolumn{3}{|l|}{ Chiroptera } \\
\hline \multicolumn{3}{|l|}{ Vespertilionidae } \\
\hline Hypsugo savii (Bonaparte, 1837) & & 10.2020, V. Ferri, P. Crescia, pers. obs. (by bat detector) \\
\hline Eptesicus serotinus (Schreber, 1774) & & 10.2020, V. Ferri, P. Crescia, pers. obs. (by bat detector) \\
\hline Miniopterus schreibersii (Kuhl, 1817) & VU & 10.2020, V. Ferri, P. Crescia, pers. obs. (by bat detector) \\
\hline \multicolumn{3}{|l|}{ Molossidae } \\
\hline Vulpes vulpes (Linnaeus, 1758) & & Amori et al., 2006 \\
\hline \multicolumn{3}{|l|}{ Mustelidae } \\
\hline Mustela nivalis Linnaeus, 1766 & $\mathrm{LC}$ & Amori et al., 2006 \\
\hline Martes foina (Erxleben, 1777) & $\mathrm{LC}$ & Amori et al., 2006 \\
\hline \multicolumn{3}{|l|}{ Artiodactyla } \\
\hline \multicolumn{3}{|l|}{ Suidae } \\
\hline Sus scrofa (Linnaeus, 1758) & $\mathrm{LC}$ & M. Perchinelli, pers. obs. \\
\hline \multicolumn{3}{|l|}{ Allochtonous species } \\
\hline \multicolumn{3}{|l|}{ Rodentia } \\
\hline \multicolumn{3}{|l|}{ Myocastoridae } \\
\hline
\end{tabular}

\section{CONCLUSION}

A total 291 vertebrates have been reported for the Palude di Torre Flavia wetland: among these 259 are native taxa, 26 allochthonous taxa and 6 belong to domestic forms. Most of the species of conservation concern are migratory birds, confirming the important role of the protected area as a stop-over site, despite its small extension.

Due to its location in a coastal area with high biological diversity, but threatened by a medium to high level of anthropization, this wetland has become a favorite site for research on ecological relationships (e.g. Fanelli et al., 2014), especially the anthropogenic impact on biodiversity and ecosystem components, and the consequent conflicts between man and wildlife (e.g. non-native plant species: Garzia et al., 2019; threat analysis and mapping: Battisti et al., 2008, 2009b; Battisti \& Santoro, 2009; Battisti et al., 2013a; marine litter and its impact: Battisti et al., 2016b, 2018c, 2019b; Poeta et al., 2014, 2015, 2016a, 2016b, 2017; Di Gennaro et al., 2019; Pietrelli et al., 2017; impacts of musical events on birds: Battisti et al., 2019a; effectiveness in wildlife management: Battisti, 2017, 2018; Battisti et al. 2013b, 2020d).

We recommend further research on the local status of the species of conservation concern in terms of local density, dynamics, and spatial distribution. Monitoring the local herpetofauna is necessary to assess the possible presence of Salamandridae, and to monitor the abundance of the endangered terrapin Emys orbicularis (Linnaeus, 1758). Among birds, further research is needed to survey a recently recorded breeding site of Nycticorax nyctirorax (Linnaeus, 1758) (Cento et al., 2017), a wintering roost of Bubulcus ibis (Linnaeus, 1758), and the effect of human disturbance on the breeding success of the locally nesting plovers (Charadrius dubius Scopoli, 1786 and $C$. alexandrinus Linnaeus, 1758; see Battisti et al., 2020f). Moreover, further studies at the ecosystem community level should be promoted (e.g., breeding bird structure and dynamics of assemblages in different habitat types: reedbeds, rushbeds, restored habitats, ecotones), also considering the role of anthropogenic and natural threats as factors of change and species turnover of these assemblages.

Finally, with regard to mammals, further studies should be aimed at quantifying the structure and dynamics of the non-native population of Myocastor coypus (Molina, 1782) and of the composition, frequency, richness and diversity of the local assemblage of Chiroptera. 


\section{Acknowledgements}

We would like to thank all colleagues who made this review possible by providing original field data, useful comments and suggestions. For fish: Massimiliano Scalici for the revision of checklist; Tonino and Sandro Mantovani (local historical fishermen), Giuseppe Moccia and Sergio Zerunian for furher information; for amphibians and reptiles: Marco A. Bologna and Vincenzo Ferri revised the check-list (nomenclature and systematic order), Luca Marini (for Caretta caretta), Riccardo Santoro, the volunteer zoophile guards (NOGRA) of Ladispoli, for their useful support in field research; for birds: C. Angelici, S. Arena, G. Benassi, M. Biondi, A. Boano, M. A. Bologna, F. Borsi, M. Brunelli, P. Bugliazzini, F. Bulgarini, T. Büttel, C. Catoni, F. Causarano, S. Ceccobelli, J.C. Cecere, A. Circosta, E. Coltellacci, E.G. Condello, M. Coppola, A. Croce, A. De Cesare, E. De Giorgi, E. De Santis, R. De Santis, E. De Zuliani, S. Di Carlo, B. Doe, A. Duiz, V. Dundee, S. Evangelisti, F. Felici, L. Fortunati, F. Foschi, B. Frank, Pa. Gennari, Pi. Gennari, P. Gianpaoletti, R. Gildi, G. Grillo, M. Grylle, M. Gustin, P. Harris, D. Iavicoli, R. La Torre, C. Lattanzi, S. Laurenti, G. Lepri Angelini, R. Lippolis, E. Lorenzetti, D. Lorica, R. Maggini, R. Malavasi, A. Manganaro, Fa. Mantero, C. Marangoni, E. Mattei, R. Mattei, G. Melchiorri, R. Molajoli, P. Monti, A. Pagotto, M. Paloni, M. Passarella, S. Patrizi, M. Perchinelli, F. Petrassi, A. Pezzarossa, L. Pietrelli, T. Pizzari, A. Polinori, G. Prola, A. Pulvirenti, G. C. Raia, E. Ranchelli, L. Redolfi De Zan, S. Rempicci, S. Riello, A. Rivola, E. Rizzo, M. Rossi, G. Santori, R. Santoro, S. Sarrocco, E. Savo, R. Scrocca, M. Sebastianelli, L. Söffker, B. Soligon, A. Sorace, G. Sozio, M. Sterpi, A. B. Tedesco, P. Tinazzo, C. Tomei, M. Trotta, D. Zacchei, D. Valenti, S. Vanadia, M. Zanini, the volunteers of the Choona! LIFE Project, and all the colleagues which participated for the first book on the biodiversity and management of this wetland (Battisti, 2006a); for mammals: Stefania Biscardi (before 2006), Vincenzo Ferri and Paolo Crescia (October 2020), carried out a field sampling to detect bats; Giovanni Amori reviewed the check-list; Francesca Marini, Christian Angelici, Simone Ceccobelli, Eva Gabrielli, Laura Montaudo, Marianna Vecchi, Riccardo Santoro and Giuseppe M. Carpaneto carried out a pilot study in the coypu; Stefano Donfrancesco and Sante Esigibili for the useful information about the case of predation by canid in the Torre Flavia wetland. We would like to acknowledge also Egidio De Angelis, Carlo Galimberti, Narciso Trucchia, operators in the Torre Flavia Natural Monument. Luigi Cicillini provided the aerial photo by drone. Two anonymous reviewers and the editor provided useful comments and suggestions.

\section{REFERENCES}

Acosta A. T. R., Jucker T., Prisco I. \& Santoro R., 2013 - Passive recovery of Mediterranean coastal dunes following limitations to human trampling. In: Restoration of coastal dunes. Martínez M. L., Gallego-Fernández J. B. \& Hesp P. A. (eds.). Springer-Verlag, Berlin: 187-198.

Aglitti C., Battisti C. \& Sorace C., 2006 - La struttura del fragmiteto. In: Biodiversità, gestione, conservazione di un'area umida del litorale tirrenico: la Palude di Torre Flavia. Battisti C. (ed.). Provincia di Roma, Assessorato alle politiche agricole e dell'ambiente, Gangemi editore, Roma: 189-190.

Amati B., Appolloni M., Quaggiotto E., Smriglio C. \& Oliverio M., 2019 - Notes on some taxa of the Alvania lineata-complex with the descriptions of three new species from the Mediterranean Sea (Gastropoda: Rissoidae). Iberus, 37 (1): 81-112.

Amati B., Trono D. \& Oliverio M., 2020 - Description of Alvania rominae n. sp. from the Mediterranean Sea (Mollusca Gastropoda Rissoidae). Biodiversity Journal, 11 (2): 541-551.

Amori G. \& Battisti C., 2008 - An invaded wet ecosystem in central Italy: an arrangement and evidence for an alien food chain. Rendiconti Lincei, 19 (2): 161-171.

Amori G., Battisti C. \& Capizzi D., 2006 - Mammiferi. In: Biodiversità, gestione, conservazione di un'area umida del litorale tirrenico: la Palude di Torre Flavia. Battisti C. (ed.). Provincia di Roma, Assessorato alle politiche agricole e dell'ambiente, Gangemi editore, Roma: 325-326.

Amori G., Contoli L. \& Nappi A., 2008 - Mammalia II - Erinaceomorpha, Soricomorpha, Lagomorpha, Rodentia. Fauna d'Italia, vol. 44. Edizioni Calderini - Il Sole 24 Ore, Bologna.

Amori G., Battisti C. \& De Felici S., 2009 - I Mammiferi della Provincia di Roma. Dallo stato delle conoscenze alla gestione e conservazione delle specie. Provincia di Roma, Assessorato alle politiche dell'agricoltura, Stilgrafica, Roma.

Angelici C., Marini F., Battisti C., Bertolino S., Capizzi D. \& Monaco A., 2012 - Cumulative impact of rats and coypu on nesting waterbirds: first evidences from a small Mediterranean wetland (central Italy). Vie et Milieu - Life and Environment, 62: 137-141.

Anzalone B., Iberite M. \& Lattanzi E., 2010 - La flora vascolare del Lazio. Informatore Botanico Italiano, 42 (1): 187-317.

Arena S., Battisti C. \& Carpaneto G. M., 2009 - Uso degli ambienti umidi (giuncheti, fragmiteti) da parte della Passera d'Italia Passer italiae: dati preliminari. Ecologia urbana, 21: 63-65.

Arena S., Battisti C. \& Carpaneto G. M., 2011 - The ecological importance of wetlands for aerial insectivores (swifts, martins and swallows) along the Tyrrhenian coast. Rendiconti Lincei, 22: 395-402.

Baccetti N., Fracasso G. \& Commissione Ornitologica Italiana, 2019 La nuova Lista CISO-COI degli uccelli italiani 2019. Centro Italiano Studi Ornitologici. <https://ciso-coi.it/coi/checklist-ciso-coidegli-uccelli-italiani/>

Bartolucci F. \& Iocchi M., 2007 - Note floristiche per il Lazio. Informatore Botanico Italiano, 39: 35-38.

Battisti C. (ed.), 2006a - Biodiversità, gestione, conservazione di un'area umida del litorale tirrenico: la Palude di Torre Flavia. Provincia di Roma, Assessorato alle politiche agricole e dell'ambiente, Gangemi editore, Roma.

Battisti C., 2006b - Ciclo annuale delle comunità ornitiche: un'analisi con il metodo del transetto. In: Biodiversità, gestione, conservazione di un'area umida del litorale tirrenico: la Palude di Torre Flavia. Battisti C. (ed.). Provincia di Roma, Assessorato alle politiche agricole e dell'ambiente, Gangemi editore, Roma: 270-280.

Battisti C., 2007 - Falco di palude, Circus aeruginosus, Anatidi e profondità dell'acqua in un'area umida costiera dell'Italia centrale. $R i$ vista Italiana di Ornitologia - Research in Ornithology, 77: 81-90.

Battisti C., 2014a - Check-list of Vertebrates in the "Tenuta dei Massimi" nature reserve (Rome, central Italy) with some remarks on local conservation priorities. Natural History Sciences, 1: 25-36.

Battisti C., 2014b - Bird assemblages on a Mediterranean sandy beach: a yearly study. Rivista Italiana di Ornitologia - Research in Ornithology, 84 (1): 5-10.

Battisti C., 2016 - Experiential key species for the nature-disconnected generations. Animal Conservation, 19: 485-487.

Battisti C., 2017 - How to make (in) effective conservation projects: look at the internal context! Animal Conservation, 20: 305-307.

Battisti C., 2018 - Why is it so difficult to have success? Applying the Swiss Cheese theory to environmental practices. Environmental Practice, 20: 42-54.

Battisti C., 2020 - Sepia cuttlebones pecked by birds along a Mediterranean beach: patterns, frequency and a possible conservation implication. Avocetta, 44: 95-99.

Battisti C. \& Buccomino G., 1999 - I Siti di Importanza Comunitaria costieri della Provincia di Roma. Quaderni IAED, 12: 94-112. 
Battisti C. \& Santoro R., 2009 - Approccio expert-based per l'analisi di un sito. Estimo e Territorio, 1: 25-29.

Battisti C. \& Sorace A., 2005 - Piscicoltura, apporto idrico artificiale e regolazione delle acque: individuazione dei periodi chiave per la conservazione dell'avifauna in un'area umida residuale. In: Atti XIII Convegno Italiano di Ornitologia. Boano G., Cucco M., Pavia M. \& Rubolini D. (eds.). Avocetta, 29: 39.

Battisti C. \& Sorace A., 2006 - Uccelli: check-list aggiornata al 2005. In: Biodiversità, gestione, conservazione di un'area umida del litorale tirrenico: la Palude di Torre Flavia. Battisti C. (ed.). Provincia di Roma, Assessorato alle politiche agricole e dell'ambiente, Gangemi editore, Roma: 255-269.

Battisti C. \& Zocchi A., 2018 - Experiential key species for naturedisconnected generations: an expert-based framework for their apriori selection. Anthrozoos, 31: 627-644.

Battisti C., Sorace A., De Angelis E., Galimberti C. \& Trucchia N., 2004 - Waterbird abundance in a residual wetland of Central Italy during two years of contrasting water level. Avocetta, 28: 86-90.

Battisti C., Sorace A., De Angelis E., Galimberti C. \& Trucchia N., 2005 - Ciclo biennale di Ardeidi, Anatidi, Rallidi nella palude di Torre Flavia (Roma, Italia centrale). Rivista Italiana di Ornitologia - Research in Ornithology, 75: 3-16.

Battisti C., Sorace A., De Angelis E., Galimberti C. \& Trucchia M., 2006 - Ciclo biennale di ardeidi, anatidi, rallidi. In: Biodiversità, gestione, conservazione di un'area umida del litorale tirrenico: la Palude di Torre Flavia. Battisti C. (ed.). Provincia di Roma, Assessorato alle politiche agricole e dell'ambiente, Gangemi editore, Roma: 281-286.

Battisti C., Luiselli L., Pantano D. \& Teofili C., 2008 - On threats analysis approach applied to a Mediterranean remnant wetland: Is the assessment of human-induced threats related to different level of expertise of respondents? Biodiversity and Conservation, 17 (6): 1529-1542.

Battisti C., Malavasi R. \& Carpaneto G. M., 2009a - Breeding and wintering bird assemblages in a mediterranean wetland: a comparison using a diversity/dominance approach. Vie et Milieu - Life and Environment, 59: 1-6.

Battisti C., Luiselli L. \& Teofili C., 2009b - Quantifying threats in a Mediterranean wetland: are there any changes in their evaluation during a training course? Biodiversity and Conservation, 18: 30533060 .

Battisti C., Biondi M. \& Pietrelli L., 2011 - Il calpestio come disturbo alla nidificazione del Corriere piccolo (Charadrius dubius) e del Fratino (Charadrius alexandrinus). Dati preliminari sull'impatto in un sito del Litorale Romano. In: Il Fratino: status, biologia e conservazione di una specie minacciata. Biondi M. \& Pietrelli L. (eds.). Atti del convegno nazionale, Bracciano (RM), 18 settembre 2010. Edizioni Belvedere, Latina: 55-60.

Battisti C., Luiselli L. \& Vignoli L., 2012a - Bird assemblages in a structurally simplified Mediterranean sandy beach: an analysis at spatial and temporal level. Revue d'Ecologie (Terre Vie), 67: 63-70.

Battisti C., Circosta A., De Angelis E., Evangelisti F., Fraticelli F., Galimberti C., Hueting S. \& Trucchia N., 2012b - La Moretta tabaccata Aythya nyroca in una zona umida mediterranea (Palude di Torre Flavia, Italia centrale): fenologia locale su base pluriennale (20032011). Alula, 19: 185-187.

Battisti C., Caffari A., Carbone F., Castellaccio F., Ciambotta M., Cucchi F., Di Pasquale D., Ferracuti C., Grassi F., Marini F., Serrani S. \& Spoletini A., 2013a - Minacce di origine antropica in un'area protetta dell'Italia centrale. Territori, 13: 34-41.

Battisti C., Franco D. \& Luiselli L., 2013b - Searching the conditioning factors explaining the (in)effectiveness of protected areas management: A case study using a SWOT approach. Environmental Practice, 15 (4): 401-407.

Battisti C., Boano A., Cento M., Circosta A. \& Muratore S., 2015a Waders (Aves, Charadriiformes) in a Mediterranean remnant wetland: a year-round pilot study evidences contrasting patterns in diversity metrics. Rivista Italiana di Ornitologia - Research in Ornithology, 85: 61-66.

Battisti C., Marini F. \& Vignoli L., 2015b - A five-year cycle of coypu abundance in a remnant wetland: a case of sink population collapse? Hystrix, the Italian Journal of Mammalogy, 26: 37-40.
Battisti C., Boano A., Monaco E., Muratore S., Mazzarani D., De Zuliani E., Demartis P., Piroli R. \& Scrocca R., 2016a - Ciclo annuale delle comunità ornitiche in due zone umide costiere del Lazio (Palude di Torre Flavia e Macchiatonda, Italia centrale). Alula, 23: 3-13.

Battisti C., Poeta G., Pietrelli L. \& Acosta A. T. R, 2016b - An unexpected consequence of plastic litter clean-up on beaches: too much sand might be removed. Environmental Practice, 18: 242-246.

Battisti C., Frank B. \& Fanelli G., 2018a - Children as drivers of change: The operational support of young generations to conservation practices. Environmental Practice, 20: 129-135.

Battisti C., Fanelli G., Bertolino S., Luiselli L., Amori G. \& Gippoliti S., $2018 \mathrm{~b}$ - Non-native invasive species as paradoxical ecosystem services in urban conservation education. Web Ecology, 18: 37-40.

Battisti C., Malavasi M. \& Poeta G., 2018c - Applying diversity metrics to plastic litter 'communities': a first explorative and comparative analysis. Rendiconti Lincei, 29: 811-815.

Battisti C., Marini F., Sarrocco S., De Santis E. \& Savo E., 2019a - Analisi degli impatti di un evento musicale (Jova Beach Party, Campo di Mare, Italia centrale) su comunità ornitiche urbane e di mosaico ambientale: prime evidenze. Alula, 26 (1-2): 15-24.

Battisti C., Kroha S., Kozhuharova E., De Michelis S., Fanelli G., Poeta G., Pietrelli L. \& Cerfolli F., 2019b - Fishing lines and fish-hooks as neglected marine litter: first data on chemical composition, densities, and biological entrapment from a Mediterranean beach. Environmental Science and Pollution Research, 26: 1000-1007.

Battisti C., Grosso G., Ioni S., Zullo F. \& Cerfolli F., 2020a - Response of specialized birds to red-bed aging in a Mediterranean wetland: significant changes in bird biomass after two decades. Israel Journal of Ecology and Evolution. <https://doi.org/10.1163/22244662bja10007>

Battisti C., Dodaro G. \& Vannuccini M., 2020b - A fine-grained bird Atlas as tool for spatial monitoring: a case study from a remnant wetland during the breeding period (Torre Flavia, central Italy). Rivista Italiana di Ornitologia - Research in Ornithology, 90 (1): 41-50.

Battisti C., De Angelis E., Galimberti C. \& Trucchia N., 2020c - La gestione operativa di un ecosistema: la Palude di Torre Flavia. Un manuale per studenti e volontari. Città Metropolitana di Roma Capitale, Roma.

Battisti C., Fanelli G., Marini F., Amori G. \& Luiselli L., 2020d - Assessing the nature reserve management effort using an expertbased threat analysis approach. Diversity, 12: 145. < doi:10.3390/ d12040145>

Battisti C., Cento M., Circosta A., Coppola M., Fraticelli F., Grillo G. \& Muratore S., 2020e - Il Fistione turco (Netta rufina) nella Palude di Torre Flavia (Italia centrale): apparente incremento della presenza occasionali. Alula, 27 (1-2): 82-85.

Battisti C., Gustin M. \& Polinori A., 2020f - Do the weaknesses and strengths of experts and local volunteers affect the conservation actions focused on nesting plovers? Constructive considerations from the Italian beach front line. Avocetta, 44: 105-111.

Benassi G. \& Battisti C., 2009 - Water level decrease and change in abundance of water-obligate bird species: preliminary data from a remnant wetland of central Italy. Aldrovandia, 5: 229-234.

Benassi G. \& Battisti C., 2011 - Frequency of occurrence of a set of water-related bird species in an archipelago of remnant marshlands of Central Italy. Rendiconti Lincei, 22: 11-16.

Benassi G., Battisti C. \& Luiselli L., 2007 - Area effect on bird species richness of an archipelago of wetland fragments of Central Italy. Community Ecology, 8: 229-237.

Benassi G., Battisti C., Luiselli L. \& Boitani L., 2009 - Area-sensitivity of three reed bed bird species breeding in Mediterranean marshland fragments. Wetlands Ecology Management, 17: 555-564.

Bentivegna F., Rasotto M. B., De Lucia G. A., Secci E., Massaro G., Panzera S., Caputo C., Carlino P., Treglia G. \& Hochscheid S., 2010 - Loggerhead turtle (Caretta caretta) nests at high latitudes in Italy: a call for vigilance in the Western Mediterranean. Chelonian Conservation and Biology, 9: 283-289.

Bertolino S., 2016 - Using native experiential key species to avoid exotic species filling the emotional void: response to Battisti's 'Letter from the Conservation Front Line'. Animal Conservation, 19: 488-489. 
Bertoni R., 2012 - La Rete Italiana per la ricerca ecologica a lungo termine (LTER-Italia). Aracne Editrice, Roma.

Biondi M., Pietrelli L. \& Guerrieri G., 1992 - Avvistamenti di particolare interesse sul Litorale Romano. Picus, 18: 141-147.

Biondi M. \& Pietrelli L., 1999 - Trend negativo della nidificazione di Corriere piccolo, Charadrius dubius, in aree costiere del Lazio. Rivista Italiana di Ornitologia - Research in Ornithology, 69 (2): 161-167.

Biondi M. \& Pietrelli L., 2009 - Annexing by Kentish Plover Charadrius alexandrinus of nest and egg of Little Ringed Plover Charadrius dubius in Latium (central Italy). Uccelli d'Italia, 34: 133-134.

Biondi M., Corso A. \& Grussu M., 2004 - Nuovi dati sullo svernamento e la fenologia del Calandro maggiore Anthus richardi in Italia (1995-2004). Gli Uccelli d'Italia, 29: 39-47.

Biondi M., De Vita S., Pietrelli L., Muratore S., De Giacomo U., Valenti D. \& Landucci G., 2014 - Monitoraggio riproduttivo delle popolazioni costiere di Fratino Charadrius alexandrinus e Corriere piccolo Charadrius dubius nel Lazio (2014). Gli Uccelli d'Italia, 39: 35-40.

Biondi M., Prola G. \& Stanislavovich P. P., 2016 - Svernamento nel Lazio (Italia centrale) di un Fratino Charadrius alexandrinus inanellato in Ucraina. Gli Uccelli d'Italia, 41: 143-144.

Biondi M., Pietrelli L. \& Muratore S., 2017 - Nuovi dati sullo svernamento del Fratino Charadrius alexandrinus nel Lazio (2016-17). Gli Uccelli d'Italia, 42: 5-15.

Biondi M., Pietrelli L., Menegoni P. \& Muratore S., 2020 - Il Fratino Charadrius alexandrinus nel Lazio: periodo 2014-2020. Gli Uccelli d'Italia, 45: 116-126.

BirdLife International, 2017- European birds of conservation concern: populations, trends and national responsabilities. BirdLife International, Cambridge, UK.

Blasi C. \& Michetti L., 2005 - Biodiversità e Clima. In: Stato della biodiversità in Italia. Contributo alla strategia nazionale per al biodiversità. Blasi C, Boitani L., La Posta S., Manes F. \& Marchetti M. (eds.). Ministero dell'Ambiente e della Tutela del territorio. F.lli Palombi editori, Roma.

Blondel J. \& Aronson J., 1999 - Biology and wildlife of the Mediterranean region. Oxford University Press, USA.

Boitani L., Lovari S. \& Vigna Taglianti A., 2003 - Mammalia III - Carnivora - Artiodactyla. Fauna d'Italia, vol., 38. Edizioni Calderini - Il Sole 24 Ore, Bologna.

Bologna G., Petretti F. \& Vigna-Taglianti A., 1974 - Sul Falco pescatore Pandion haliaetus e sul Mignattaio Plegadis falcinellus. Rivista Italiana di Ornitologia - Research in Ornithology, 44: 153-154.

Bologna M. A., Capula M. \& Carpaneto G. M. (eds.), 2000 - Anfibi e rettili del Lazio. F.lli Palombi editori, Roma.

Bologna M. A., Salvi D. \& Pitzalis M. (eds.), 2007 - Atlante degli anfibi e rettili della Provincia di Roma. Provincia di Roma, Assessorato alle Politiche agricole e ambientali, Università degli studi Roma Tre, Roma.

Brichetti P. \& Fracasso G., 2015 - Check-list degli uccelli italiani aggiornata al 2014. Rivista Italiana di Ornitologia - Research in Ornithology, 85 (1), 31-50.

Brichetti P., Arcamone E. \& C.O.I., 1998 - Comitato di Omologazione italiano (C.O.I.). 13. Rivista Italiana di Ornitologia - Research in Ornithology, 68: 205-208.

Britton R. H. \& Crivelli A. J., 1993 - Wetlands of southern Europe and North Africa: mediterranean wetlands. In: Wetlands of the World, vol. 1. Whigham D. F., Dykyjova D. \& Hejny S. (eds.). Kluwer Academic Publishers, Dordrecht: 129-195.

Brunelli M. \& Fraticelli F., 2010 - Gli uccelli di comparsa accidentale nel Lazio: aggiornamento a tutto il 2009. Alula, 17: 23-42.

Brunelli M. \& Sorace A., 1999 - Avvistamenti e comportamenti insoliti. Alula, 6: 179-184.

Brunelli M. \& Sorace A., 2000 - Avvistamenti e comportamenti insoliti. Alula, 7: 88-92.

Brunelli M. \& Sorace A., 2001 - Avvistamenti e comportamenti insoliti. Alula, 8: 101-106.

Brunelli M., Calvario E., Fraticelli F., Sarrocco S. \& Gibertini G., 2001 Il catalogo della Collezione ornitologica di Palazzo Ruspoli di Cerveteri (Roma). Alula, 8: 3-28.
Brunelli M., Sarrocco S., Corbi F., Sorace A., Boano A., De Felici S., Guerrieri G., Meschini A. \& Roma S., 2011 - Nuovo Atlante degli Uccelli Nidificanti nel Lazio. Edizioni ARP (Agenzia Regionale Parchi), Roma.

Buccomino G. \& Leporatti M. L., 2009 - Contributo alla conoscenza della flora vascolare del Monumento naturale Palude di Torre Flavia (Lazio). Informatore Botanico Italiano, 41 (2), 325-341.

Carpaneto G. M., Mazziotta A. \& Vigna Taglianti A., 2006 - Ricerche preliminari sui Coleotteri. In: Biodiversità, gestione e conservazione di un'area umida del litorale tirrenico: la Palude di Torre Flavia. Battisti C. (ed.). Provincia di Roma, Assessorato alle politiche agricole e dell'ambiente, Gangemi Editore, Roma, 205-214.

Causarano F. \& Battisti C., 2009 - Effect of seasonal water level decrease on a sensitive bird assemblage in a Mediterranean wetland. Rendiconti Lincei, 20: 211-218.

Causarano F., Battisti C. \& Sorace A., 2006 - Censimento dell'avifauna nidificante con il metodo del mappaggio: anadamento quinquennale ed effetti dello stress idrico. In: Biodiversità, gestione, conservazione di un'area umida del litorale tirrenico: la Palude di Torre Flavia. Battisti C. (ed.). Gangemi editore, Provincia di Roma, Assessorato alle politiche agricole e dell'ambiente, Roma: 306-315.

Causarano F., Battisti C. \& Sorace A., 2009 - Effect of winter water stress on the breeding bird assemblage of a remnant wetland in Central Italy. Revue d'Écologie (Terre Vie), 64: 61-72.

Ceccobelli S. \& Battisti C., 2010 - On the water depth in diving sampling sites of Tachybaptus ruficollis. Rendiconti Lincei, 21: 359364.

Celauro D., 2006 - Pesci: dati preliminari. In: Biodiversità, gestione, conservazione di un'area umida del litorale tirrenico. Battisti C (ed.). Provincia di Roma, Assessorato alle politiche agricole e dell'ambiente, Gangemi editore, Roma: 234-239.

Cento M., Giampaoletti P., Grillo G., Circosta A., Coppola M. \& Battisti C., 2017 - Nidificazione di Nitticora Nycticorax nycticorax e note sul roost di ardeidi nel Monumento naturale Palude di Torre Flavia (Lazio, Italia centrale). Alula, 24: 124-126.

Chiesa S., 2006 - Il gambero rosso della Louisiana (Procambarus clarkii Girard, 1852) nel Monumento naturale "Palude di Torre Flavia. In: Biodiversità, gestione, conservazione di un'area umida del litorale tirrenico. Battisti C. (ed.). Provincia di Roma, Assessorato alle politiche agricole e dell'ambiente, Gangemi editore: 354-359.

Chiesa S., Scalici M. \& Gibertin G., 2006 - Occurrence of allochthonous freshwater crayfishes in Latium (Central Italy). Bulletin Français de la Pêche et de la Pisciculture, 380-381: 883-902.

Condello E. G., 2018 - Revisione e aggiornamento delle conoscenze sulla presenza del Sacro Falco cherrug nel Lazio. Alula, 25: 37-46.

Conigliaro M. \& Battisti C., 2011 - Andamenti giornalieri e intrastagionali invernali di Folaga Fulica atra e anatidi in un'area umida del litorale tirrenico (Palude di Torre Flavia, Italia centrale). Alula, 18: 17-31.

Conigliaro M., Battisti C., Amori G. \& Luiselli L., 2011 - Diving times and pecking rates of the Eurasian Coot (Fulica atra) in different habitat types: a pilot study. Rendiconti Lincei, 22: 47-53.

Corti C., Capula M., Luiselli L., Razzetti E. \& Sindaco R., 2011 - Fauna d'Italia. Vol. XLV. Reptilia. Edizioni Calderini - Il Sole 24 ORE S.p.A., Bologna.

Crucitti P., Amori G., Battisti C. \& Giardini M., 2013 - Check-list degli Anfibi, Rettili, Uccelli e Mammiferi dell'area "arcipelago mentanese-cornicolano" (Campagna Romana, Lazio). Bollettino Museo Civico di Storia Naturale di Verona, Botanica Zoologia, 37: 29-46.

Crucitti P., Brocchieri D., Cervoni F., Di Russo E., Giardini M., Manetti C. \& Santoboni L., 2019 - Checklist dei Vertebrati del Parco Naturale Archeologico dell'Inviolata (Guidonia Montecelio, Roma). Quaderni del Museo Civico di Storia Naturale di Ferrara, 7: 6179.

Davidson N.C., Fluet-Chouinard E. \& Finlayson C.M., 2018 - Global extent and distribution of wetlands: trends and issues. Marine and Freshwater Research, 69: 620-627.

Davoli L., Raffi R., Baldassarre M. A., Bellotti P. \& Di Bella L., 2019 New maps relative to the Special Protection Area of the "Palude di Torre Flavia" (central Tyrrhenian sea, Italy) prone to severe coastal erosion. Italian Journal of Engineering Geology and Environment, 2. <doi: 10.4408/IJEGE.2019-02.O-01> 
De Giovanni A., 2015 - Gli Anticidi della Collezione Meloni nel Museo civico di storia naturale "G. Doria" di Genova. Annali del Museo Civico di Storia Naturale "G. Doria”, 107: 337-359.

Dell'Anna L. \& Avellinese E., 2006 - Odonati: dati preliminari. In: Biodiversità, gestione, conservazione di un'area umida del litorale tirrenico: la Palude di Torre Flavia. Battisti C. (ed.). Provincia di Roma, Assessorato alle politiche agricole e dell'ambiente, Gangemi editore, Roma: 202-204.

Della Bella V., Puccinelli C. \& Mancini L., 2006 - Diatomee. In: Biodiversità, gestione, conservazione di un'area umida del litorale laziale: la Palude di Torre Flavia. Battisti C. (ed.). Provincia di Roma, Assessorato alle politiche agricole e dell'ambiente, Gangemi editore, Roma: 151-163.

Della Bella V., Puccinelli C., Marcheggiani S. \& Mancini L., 2007 Benthic diatom communities and their relationship to water chemistry in wetlands of central Italy. Annales de Limnologie-International Journal of Limnology, 43: 89-99.

Del Vecchio S., Porceddu M., Fantinato E., Acosta A. T., Buffa G. \& Bacchetta G., 2018 - Germination responses of Mediterranean populations of Cakile maritima to light, salinity and temperature. Folia Geobotanica, 53 (4), 417-428.

Di Bella L., Raffi R., Alivernini M., Baldassarre M. A., Barbieri M., Bellotti P., Biancone M., Calise G., D’Orefice M., Frenzel P., Frezza V., Tarragoni C. \& Davoli L., 2020 - Coastal morphodynamics and environmental assessment of the Special Protection Site of Palude di Torre Flavia (Tyrrhenian Sea, Italy). Environmental Earth Sciences, 79 (514). <https://doi.org/10.1007/s12665-020-09257-z>

Di Blasio L., Santoro R., Ferri V., Battisti C., Soccini C., Egidi A. \& Scalici M., in press - First successful reproduction of the Chinese Striped-necked Turtle Mauremys sinensis (Gray, 1834) in a remnant wetland of central Italy. BioInvasion Records.

Di Gennaro A., Pietrelli L., Iannilli V., Lecce F., Battisti C., Acosta A. T. R. \& Poeta G., 2019 - Beach Litter: prime evidenze di nuovi impatti in ambiente costiero, il caso delle egagropili di fanerogame marine. Atti Convegni Lincei, 328: 101-105.

Di Giovanni F. \& Reshchikov A., 2016 - Contribution to the knowledge of Ichneumonidae (Hymenoptera) in Italy. Linzer biologische Beiträge, 48 (1): 495-505.

Dioli P., 2010 - Presenza in Toscana e all'Elba del ligeide tropicale Graptostethus servus (Fabricius, 1787) e altre nuove segnalazioni nel Lazio e in Campania (Hemiptera, Heteroptera, Lygaeidae). Onychium, 8: 6-10.

Di Tucci F. \& Chigi F., 1937 - Paludi regolate sul litorale del medio Tirreno. Pinci Editore, Roma.

Fabrini G., 2019 - Seed germination data of Sporobolus aculeatus (Poaceae) and Juncus subulatus (Juncaceae). Flora, 29: 303.

Fanelli G., 2006 - Dinamica e disturbo nella vegetazione palustre di Torre Flavia: fondamenti per una gestione naturalistica. In: Biodiversità, gestione, conservazione di un'area umida del litorale tirrenico. Battisti C. (eds.). Provincia di Roma, Assessorato alle politiche agricole e dell'ambiente, Gangemi editore, Roma: 388-391.

Fanelli G. \& Bianco P. M. (eds.), 2007 - Memoria illustrativa della carta della vegetazione della Provincia di Roma. Provincia di Roma, Dip. VI - Governo del territorio, Serv. 3. Sistema Informativo Geografico, Roma.

Fanelli G., D'Angelis D., De Sanctis M. \& Serafini Sauli A., 2011 - Notule floristiche per il Lazio. Informatore Botanico Italiano, 43: 171-172.

Fanelli G., Battisti C. \& Malavasi R., 2014 - Comparing alpha-diversity between plants and birds in a remnant wetland: evidence for a threshold and implication for management. Wetlands Ecology and Management, 22: 565-569.

Fatucci M. \& Cerilli E., 2015 - Gli uccelli nel castello di Santa Severa (Roma) durante il Basso Medioevo (XIII - XIV secolo): significato paleoeconomico e ambientale, dati preliminari. Annali dell'Università degli Studi di Ferrara, Museologia Scientifica e Naturalistica, 11: 123-126.

Ferri V., Grano M., Marini D., Soccini C., Filippi E., Cervoni F. \& Masulli A., 2019 - La biodiversità aliena dei Cheloni nel Lazio: monitoraggio dei nuclei di testuggini esotiche terrestri e palustri introdotti negli ambienti naturali della regione. In: Atti II Congresso Nazionale Testuggini e Tartarughe (Albenga, 11-13 aprile 2019). Ottonello D., Oneto F., Piccardo P., Salvidio S. (eds.).: 107-113.
Ferri V., Battisti C., Soccini C. \& Santoro R., 2020 - A hotspot of xenodiversity: First evidence of an assemblage of non-native freshwater turtles in a suburban wetland in Central Italy. Lakes and Reservoir, 25: 250-257.

Ferri V., Battisti C., Soccini C., \& Santoro R., 2021 - First records for Europe of the non-native turtles Kinosternon subrubrum Bonnaterre, 1789 and Pelomedusa olivacea (Schweigger, 1812) in a suburban wetland in central Italy. Herpetology Notes, 14: 303-307.

Ficetola F. G., Siesa M. E., Manenti R., Bottoni L., De Bernardi F. \& Padoa-Schioppa E., 2011 - Early assessment of the impact of alien species: differential consequences of an invasive crayfish on adult and larval amphibians. Diversity and Distributions, 17 (6): 11411151.

Fortunati L. \& Battisti C., 2008 - Comunità ornitiche nidificanti lungo un gradiente di urbanizzazione in un mosaico ambientale dell'Italia centrale (Aves). Aldrovandia, 4: 105-110.

Fortunati L \& Battisti C., 2011 - Diving times and feeding rate by pecking in the Eurasian coot (Fulica atra). Ethology Ecology \& Evolution, 23: 165-170.

Fraticelli F., 1997 - Una Ballerina nera, Motacilla alba yarrellii, nel Lazio. Rivista Italiana di Ornitologia - Research in Ornithology, 67: 99-100.

Fraticelli F., 1998 - Avvistamento di un Piro piro pettorossiccio Limnodromus scolopaceus. Alula 5: 156-157.

Fraticelli F., 2014 - Comportamento aggressivo eterospecifico in Piro piro piccolo Actitis hypoleucos. Gli Uccelli d'Italia, 39: 100-101.

Fraticelli F. \& Prola G., 1996 - Specie interessanti presenti nella collezione ornitologica Prola. Alula, 3: 133-134.

Fraticelli F., Monaco E., Muratore S., Savalli V. \& Scarfò F., 2016 L'avifauna della Riserva naturale di Macchiatonda (Rm): check list e analisi comparativa con altre zone umide del litorale tirrenico. Gli Uccelli d'Italia, 41: 173-190.

Gachelin G., Garner P., Ferroni E., Verhave J. P. \& Opinel A., 2018 Evidence and strategies for malaria prevention and control: a historical analysis. Malaria journal, 17 (1): 1-18.

Garzia M., Iacobelli L., Scalici M., Fanelli G., D’Angeli A., Gregori G., Guidobaldi G., Marengo L. \& Battisti C., 2019 - Aliens come from the edge: a distribution pattern of focal alien plants in a small coastal reserve. Quaderni del Museo Civico di Storia Naturale di Ferrara, 7: 113-119.

Giannuzzi-Savelli R., Pusateri F., Prkić J., Bartolini S., Russini V., Fassio G. \& Oliverio M., 2020 - Revision of Mediterranean and NE Atlantic Raphitomidae (Gastropoda, Conoidea) 8: The genus Leufroyia Monterosato, 1884. Zoosystema, 42: 433-473.

Gramegna C., Della Bella V., Vigliotti F. \& Mancini L., 2006 - Macrobentofauna acquatica. In: Biodiversità, gestione, conservazione di un'area umida del litorale tirrenico. Battisti C. (ed.). Provincia di Roma, Assessorato alle politiche agricole e dell'ambiente, Gangemi editore, Roma: 230-233.

Grillo G., Sartori G., Battisti C., Ferri V., Luiselli L., Amori G. \& Carpaneto G. M., 2020 - Attempted copulatory behaviour between two phylogenetically unrelated alien species (Coypu, Myocastor coypus, and Pond slider, Trachemys scripta): first evidence. Zoology and Ecology, 30. < doi.org/10.35513/21658005.2020.2.10>

Guidi A., 2006 - Introduzione alla flora e alle comunità vegetali. In: Biodiversità, gestione e conservazione di un'area umida del litorale tirrenico: la Palude di Torre Flavia. Battisti C. (ed.). Provincia di Roma, Assessorato alle politiche agricole e dell'ambiente, Gangemi editore, Roma: 169-187.

Gustin M., Nardelli R., Brichetti P., Battistoni A., Rondinini C. \& Teofili C. (eds.), 2019 - Lista Rossa IUCN degli uccelli nidificanti in Italia 2019. Comitato Italiano IUCN e Ministero dell'Ambiente e della Tutela del Territorio e del Mare, Roma.

Iannilli V., Di Gennaro A., Lecce F., Sighicelli M., Falconieri M., Pietrelli L., Poeta G. \& Battisti C., 2018 - Microplastics in Talitrus saltator (Crustacea, Amphipoda): new evidence of ingestion from natural contexts. Environmental Science and Pollution Research, 25: 28725-28729.

Ioni S., Battisti C. \& Fanelli G., 2020 - Mapping vegetation dynamics on embryonic sand dunes: a fine-grained atlas for periodic plant monitoring in a Mediterranean protected area. Quaderni del Museo Civico di Storia Naturale di Ferrara, 8: 37-42. 
Janni O. \& Fracasso G. (eds.), 2012 - Commissione Ornitologica Italiana (COI). Report 24. Avocetta, 36: 81-87.

Lanza B., 2012 - Mammalia V - Chiroptera. Fauna d'Italia, vol. 47. Edizioni Calderini - Il Sole 24 Ore, Bologna.

Lanza B., Andreone F., Bologna M. A., Corti C. \& Razzetti E. (eds.), 2007 - Amphibia. Fauna d'Italia 42. Calderini, Bologna.

Laurenti S., 2002 - Prima cattura di Cannaiola di Jerdon Acrocephalus agricola nel Lazio. Alula, 9: 104-105.

Lorenzetti E. \& Taffon D., 2007 - Analisi delle comunità ornitiche nidificanti. In: Materiali per la conservazione delle aree umide residuali del litorale romano. Battisti C., Della Bella V. \& Guidi A. (eds.). Provincia di Roma, Roma: 98-109.

Lorenzoni M., Borghesan M., Carosi A., Ciuffardi L., De Curtis O., Del Mastro G., Di Tizio L., Franzoi P., Maio G., Mojetta A., Nonnis M. F., Pizzul E., Rossi G., Scalici M., Tancioni L. \& Zanetti M., 2019 Check-list dell'ittiofauna delle acque dolci italiane. Italian Journal of Freshwater Ichthyology, 5: 239-254.

Loy A., Aloise G., Ancillotto L., Maria Angelici F., Bertolino S., Capizzi D., Castiglia R., Colangelo P., Contoli L., Cozzi B., Fontaneto D., Lapini L., Maio N., Monaco A., Mori E., Nappi A., Podestà M., Russo D., Sarà M., Scandura M. \& Amori G., 2019 - Mammals of Italy: an annotaded checklist. Hystrix, 30 (2): 87-106.

Lucchese F., 1996 - Torre Flavia. In: Ambienti di particolare interesse naturalistico del Lazio. Censimento del patrimonio vegetale del Lazio. Dinelli A. \& Guarrera P. M. (eds.). Region Lazio. Assesorato alla Cultura, Dipartimento di Biologia Vegetale, Univiversità degli Studi "La Sapienza", Roma, Quaderno 2: 70-71.

Malavasi R., Battisti C. \& Carpaneto G. M., 2006 - Distribuzione spaziale dell'avifauna nidificante in relazione alle tipologie di uso/ copertura del suolo. In: Biodiversità, gestione, conservazione di un'area umida del litorale tirrenico: la Palude di Torre Flavia. Battisti C. (ed.). Provincia di Roma, Assessorato alle politiche agricole e dell'ambiente, Gangemi editore, Roma: 316-324.

Malavasi, R., Battisti C. \& Carpaneto G. M. 2009a - Seasonal changes in bird assemblages of a remnant wetland in a cultivated Mediterranean landscape: implications for management. Ornis Hungarica, 17-18: 25-33.

Malavasi R., Battisti C. \& Carpaneto G. M., 2009b - Seasonal bird assemblages in a Mediterranean patchy wetland: corroborating the intermediate disturbance hypothesis. Polish Journal of Ecology, 57: 171-179.

Marini F., Battisti C., Ranchelli E., Ciambotta M. \& Ventura A., 2014 L'avifauna nidificante nella Riserva naturale di Monte Soratte (Lazio, Italia centrale): check-list, status di conservazione e atlante locale. Bollettino del Museo Civico di Storia Naturale di Verona (Botanica Zoologia), 38: 161-179.

Marini F., Ceccobelli S. \& Battisti C., 2011 - Coypu (Myocastor coypus) in a Mediterranean remnant wetland: a pilot study of a yearly cycle with management implications. Wetland Ecology and Management, 19: 159-164.

Marini F., Gabrielli E., Montaudo L., Vecchi M., Santoro R., Battisti C. \& Carpaneto G. M., 2013 - Diet of Coypu (Myocastor coypus) in a Mediterranean coastal wetland: a possible impact on threatened rushbeds? Vie et Milieu - Life and Environment, 63: 97-103.

Mediterranean Wetlands Observatory, 2012 - First Mediterranean Wetlands Observatory report. Technical report, 2012. Tour du Valat, France.

Mediterranean Wetlands Observatory, 2018 - Solutions for sustainable Mediterranean Wetlands, 2018. Tour du Valat, France.

Minganti A. \& Zocchi A., 2006 - Contributo alla conoscenza dei Molluschi terrestri e dulciacquicoli. In: Biodiversità, gestione, conservazione di un'area umida del litorale tirrenico: la Palude di Torre Flavia. Battisti C. (ed.). Provincia di Roma, Assessorato alle politiche agricole e dell'ambiente, Gangemi editore, Roma: 198-201.

Mingozzi T., Masciari G., Paolillo G., Pisani B., Russo M. \& Massolo A., 2007 - Discovery of a regular nesting area of loggerhead turtle Caretta caretta in southern Italy: a new perspective for national conservation. Biodiversity and Conservation, 16: 3519-3541.

Moccia G., 2006 - La pesca del novellame eurialino nel tratto di costa tirrenica della provincia di Roma con particolare riferimento alla zona della Palude di Torre Flavia. In: Biodiversità, gestione, con- servazione di un'area umida del litorale tirrenico. Battisti C. (ed.). Provincia di Roma, Assessorato alle politiche agricole e dell'ambiente, Gangemi editore, Roma: 398-400.

Molajoli R. \& Catoni C., 2001 - Oca lombardella Anser albifrons. In: Avvistamenti e comportamenti insoliti. Brunelli M., Sorace A. Alula, 8: 101-106.

Monti P., 2006 - Progetto Rondine: ricerca ed educazione ambientale. In: Biodiversità, gestione, conservazione di un'area umida del litorale tirrenico. Battisti C. (ed.). Provincia di Roma, Assessorato alle politiche agricole e dell'ambiente, Gangemi editore, Roma: 461-464.

Mori E., Menchetti M., Dondini G., Biosa D. \& Vergari S., 2014 - Theriofauna of site of community importance Poggi di Prata (Grosseto, Central Italy): terrestrial mammals and preliminary data on Chiroptera. Check List, 10 (4): 718-723.

Muratore S., Corradi A., Boano A., Capizzi D. \& Battisti C., 2015 Seasonal patterns in abundance of common aerial forager birds in two Tyrrhenian wetlands: a comparison among species and sites. Vie et Milieu - Life and Environment, 65 (3): 181-186.

Nicoletti L., Paganelli D. \& La Valle P., 2006 - Popolamenti bentonici marini tra Civitavecchia e la Foce del Tevere. In: Biodiversità, gestione, conservazione di un'area umida del litorale tirrenico: la Palude di Torre Flavia. Battisti C. (ed.). Provincia di Roma, Assessorato alle politiche agricole e dell'ambiente, Gangemi editore, Roma: 147-150.

Nimis P. L., Martellos S., Guidi A., Battisti C. \& Forti G., 2020 - Guida alle piante della Palude di Torre Flavia - Cerveteri e Ladispoli (RM). Sistema museale naturalistico Regione Lazio RE.SI.NA. Provincia di Roma - Servizio Informativo Geografico; $<\mathrm{http}: / /$ dbiodbs.units.it/carso/chiavi_pub21?sc $=422>($ Accessed November 2020).

Pagliari T., 1952 - Vecchi cacciatori. Ricordi di settanta anni di caccia. Colombo industrie grafiche, Roma.

Patrizi Montoro F., 1909 - Materiali per un'avifauna della provincia di Roma. Bollettino della Società Zoologica Italiana, 10: 1-103.

Pessolano U., Buccomino G., Villa R. \& Oliverio M., 2006 - Molluschi marini: primi dati. In: Biodiversità, gestione, conservazione di un'area umida del litorale tirrenico: la Palude di Torre Flavia. Battisti C. (ed.). Provincia di Roma, Assessorato alle politiche agricole e dell'ambiente, Gangemi editore, Roma: 191-197.

Pietrelli L. \& Biondi M., 2012 - Long term reproduction data of Kentish Plover Charadrius alexandrinus along a Mediterranean coast. Wader Study Group Bulletin, 119 (2): 114-119.

Pietrelli L., Biondi M. \& Muratore S., 2016 - Trend negativo della capacità riproduttiva del Fratino Charadrius alexandrinus nel Lazio. Gli Uccelli d'Italia, 41: 125-131.

Pietrelli L., Di Gennaro A., Menegoni P., Lecce F., Poeta G., Acosta A. T. R., Battisti C. \& Iannilli V., 2017 - Pervasive plastisphere: First record of plastics in egagropiles (Posidonia spheroids). Environmental Pollution, 229: 1032-1036.

Pitzalis M., 2007 - Erpetofauna. In: Materiali per la conservazione delle aree umide residuali del litorale romano. Battisti C., Della Bella V. \& Guidi A. (Eds.). Provincia di Roma, Roma: 83-97.

Pitzalis M. \& Bologna M. A., 2006 - Anfibi e rettili. In: Biodiversità, gestione, conservazione di un' area umida del litorale tirrenico. Battisti C (ed.). Provincia di Roma, Assessorato alle politiche agricole e dell'ambiente, Gangemi editore, Roma: 240-254.

Poeta G., Battisti C. \& Acosta.A. T. R., 2014 - Marine litter in Mediterranean sandy littorals: Spatial distribution patterns along central Italy coastal dunes. Marine Pollution Bulletin, 89: 168-173.

Poeta G., Romiti F. \& Battisti C., 2015 - Discarded bottles in sandy coastal dunes as threat for macro-invertebrate populations: first evidence of a trap effect. Vie et milieu - Life and Environment, 65 (3): 125-127.

Poeta G., Battisti C., Bazzichetto M. \& Acosta A. T. R., 2016a - The cotton bud beach: Marine litter assessment along the Tyrrhenian coast of central Italy following the marine strategy framework directive criteria. Marine Pollution Bulletin, 113: 266-270.

Poeta G., Conti L., Malavasi M., Battisti C. \& Acosta A. T. R., 2016b Beach litter occurrence in sandy littorals: The potential role of urban areas, rivers and beach users in central Italy. Estuarine, Coastal and Shelf Science, 181: 231-237. 
Pratesi F., 2006 - Un ricordo. In: Biodiversità, gestione, conservazione di un'area umida del litorale tirrenico: la Palude di Torre Flavia. Battisti C. (ed.). Provincia di Roma, Assessorato alle politiche agricole e dell'ambiente, Gangemi editore, Roma: 27-28.

Raffi R., Davoli L., Baldassarre A. M., Bellotti P., Biancone M., Calise G., Di Bella L., D’Orefice M., Frezza V. \& Tarragoni C., $2018-$ Morphodynamics of the Coastal Landscape and Environmental Aspects of Palude of Torre Flavia (Northern Latium-Italy). $20^{\text {th }}$ EGU General Assembly, EGU2018, Proceedings from the conference held 4-13 April, 2018 in Vienna, Austria.

Ranchelli E., Boccacci F., Sozio G., Mortelliti A., Battisti C. \& Boitani L., 2011 - Note sulla dimensione dei nidi e successo delle covate di cannaiola comune Acrocephalus scirpaceus in fragmiteti del litorale romano (Italia centrale). Alula, 18: 85-88.

Redolfi De Zan L., Battisti C. \& Carpaneto G. M., 2010 - Effect of spring water stress induced by fishery farming on two duck species Anas platyrhynchos L. and Anas crecca L. in a Mediterranean wetland. Polish Journal of Ecology, 58: 599-604.

Redolfi De Zan L., Battisti C. \& Carpaneto G. M., 2011 - Inter-annual and intra-seasonal patterns of abundance in a set of common waterbirds: along term study in a Mediterranean wetland. Vie et Milieu - Life and Environment, 61: 101-106.

Relini G., Tunesi L., Vacchi M., Andaloro F., D’Onghia G., Fiorentino F., Garibaldi F., Orsi Relini L., Serena F., Silvestri R., Battistoni A., Teofili C. \& Rondinini C. (eds.), 2017 - Lista Rossa IUCN dei Pesci ossei marini italiani. Comitato Italiano IUCN e Ministero dell'Ambiente e della Tutela del Territorio e del Mare, Roma. <http://www.iucn.it/pdf/Comitato IUCN Lista Rossa dei pesci ossei marini italiani 2017.pdf>

Rizzo E. \& Battisti C., 2007a - Ciclo annuale e selezione di habitat della Garzetta, Egretta garzetta, in un'area umida del litorale tirrenico: dati preliminari. Rivista Italiana di Ornitologia - Research in Ornithology, 77: 107-113.

Rizzo E. \& Battisti C., 2007b - Ciclo annuale di Anatidi e Ardeidi in un'area umida costiera in relazione alle tipologie di copertura del suolo (palude di Torre Flavia, Italia centrale). Alula, 14: 83-106.

Rizzo E. \& Battisti C., 2009 - Habitat preferences of Anatidae (Aves, Anseriformes) in a Mediterranean patchy wetland (Central Italy). Ekològia (Bratislava), 28: 66-73.

Rizzo E., Ceccobelli S. \& Battisti C., 2006 - Ricchezza e densità di ardeidi e anatidi in relazione alla tipologia di uso del suolo. In: Biodiversità, gestione, conservazione di un'area umida del litorale tirrenico: la Palude di Torre Flavia. Battisti C. (ed.). Provincia di Roma, Assessorato alle politiche agricole e dell'ambiente, Gangemi editore, Roma: 287-291.

Romano A., Montinaro G., Mattoccia M. \& Sbordoni V., 2007 - Amphibians of the Aurunci Mountains (Latium, Central Italy). Checklist and conservation guidelines. Acta Herpetologica, 2: 17-25.

Rondinini C., Battistoni A., Peronace V. \& Teofili C. (eds.), 2013 - Lista Rossa IUCN dei Vertebrati Italiani. Comitato Italiano IUCN e Ministero dell'Ambiente e della Tutela del Territorio e del Mare, Roma. <http://www. iucn.it/pdf/Comitato_IUCN_Lista_Rossa_dei_vertebrati_italiani.pdf $>$

Rotondi M., 1962 - Migratori alati. Edizioni La Rotografica Romana, Roma.

Sabia G., Petta L., Moretti F. \& Ceccarelli R., 2018 - Combined statistical techniques for the water quality analysis of a natural wetland and evaluation of the potential implementation of a FWS for the area restoration: the Torre Flavia case study, Italy. Ecological Indicators, 84, 244-253.

Santoro R., Jucker T., Prisco I., Carboni M., Battisti C. \& Acosta T. R., 2012 - Effect of trampling limitation on coastal dune plant communities. Environmental Management, 49: 534-542.

Savo E., 2002 - Segnalazione di muta eccentrica delle primarie nel Migliarino di palude, Emberiza schoeniclus. Rivista Italiana di Ornitologia - Research in Ornithology, 72 (1): 89.

Scalera R. \& Montinaro R., 2014 - Gli anfibi e rettili alloctoni del Lazio. In: Alieni. La minaccia delle specie alloctone per la biodiversità del Lazio. A. Monaco (ed.). Palombi editori, Roma: 64-73.

Scalici M., Pitzalis M. \& Gibertini G., 2009 - Crayfish distribution updating in central Italy. Knowledge and Management of Aquatic Ecosystems, 394-395: 06.

Scalici M., Chiesa S., Scuderi S., Celauro D. \& Gibertini, G., 2010 Population structure and dynamics of Procambarus clarkii (Girard,
1852) in a Mediterranean brackish wetland (Central Italy). Biological Invasions, 12 (5), 1415-1425.

Scoccianti C., 2006 - Strategie per la deframmentazione degli habitat nell'area di Torre Flavia: gli Anfibi come "specie guida". In: Biodiversità, gestione, conservazione di un'area umida del litorale tirrenico: la Palude di Torre Flavia. Battisti C. (ed.). Provincia di Roma, Assessorato alle politiche agricole e dell'ambiente, Gangemi editore, Roma: 421-432.

Sighele M. \& Janni O., 2010 - Resoconto Ornitologico Italiano. Anno 2008. Avocetta, 34: 77-92.

Sorace A., 1997 - Avvistamenti e comportamenti insoliti. Alula, 4 (1-2): 106-112.

Sorace A., Battisti C., Gustin M., Savo E., Biscontini D., Cecere J., Duiz A., Trotta M., Laurenti S., Monti P. \& Fanfani A., 2001 - Primo anno di attività della stazione di inanellamento di Torre Flavia (Ladispoli - RM). Alula, 8: 34-40.

Sorace A., Battisti C., Cecere J., Savo E., Gustin M., Laurenti S., Duiz A. \& Fanfani A., 2003 - Variazioni annuali del passaggio di migratori nel Monumento naturale "Palude di Torre Flavia" (Ladispoli, Roma). Avocetta, 27: 50.

Sorace A., Battisti C., Cecere J. G., Duiz A., Gustin M. \& Savo E., 2006 - Monitoraggio della migrazione ornitica mediante le attività di inanellamento. In: Biodiversità, gestione, conservazione di un'area umida del litorale tirrenico: la Palude di Torre Flavia. Battisti C. (ed.). Provincia di Roma, Assessorato alle politiche agricole e dell'ambiente, Gangemi editore, Roma: 292-305.

Sorace A., Battisti C., Gustin M., Rossi F., Cecere J. G., Savo E., Demartini L., Duiz A., Riello S., Iavicoli D. \& Romano C., $2010-$ Confronto tra le catture autunnali in quattro zone umide dell'Italia centrale. Alula, 17: 99-104.

Sorace A., Savo E., De Santis E., Duzi A., Iavicoli D., Riello S. \& Battisti C., 2015 - Autumn captures from Torre Flavia ringing station (Latium, central Italy) in 2001-2014. Avocetta, 39: 73-81.

Sozio G. \& Battisti C., 2009 - Comunità ornitiche nidificanti in un mosaico ambientale antropizzato (litorale laziale, Italia centrale). Ecologia Urbana, 21: 3-7.

Sozio G., Mortelliti A., Boccacci F., Ranchelli E., Battisti C. \& Boitani L., 2013 - Conservation of species occupying ephemeral and patchy habitats in agricultural landscapes: The case of the Eurasian reed warbler. Landscape and Urban Planning, 119: 9-19.

Speybroeck J., Beukema W., Dufresnes C., Fritz U., Jablonski D., Lymberakis P., Martínez-Solano I., Razzetti E., Vamberger M., Vences M., Vörös J. \& Crochet P.-A., 2020 - Species list of the European herpetofauna. 2020 update by the Taxonomic Committee of the Societas Europaea Herpetologica. Amphibia-Reptilia, 41: 139-189.

Sutherland W. J., 2008 - The conservation handbook: research, management and policy. John Wiley \& Sons, New York.

Talbi A., Samraoui F., Samraoui B., Zullo F. \& Battisti C., 2020 - Habitat selection of Coot (Fulica atra) and Moorhen (Gallinula chloropus) in a remnant Mediterranean wetland (Italy): Implications for conservation. Lakes and Reservoir: Research and Management. <DOI: $10.1111 /$ lre.12347>

Vannini A., Bruni G., Ricciardi G., Platania L., MoriE. \& Tricarico E., 2018 Gambusia holbrooki, the 'tadpolefish'. The impact of its predatory behaviour on four protected species of European amphibians. Aquatic Conservation: Marine and Freshwater Ecosystems, 28: 476-484.

Webb C. \& Joss J., 1997 - Does predation by the fish Gambusia holbrooki (Atheriniformes: Poeciliidae) contribute to declining frog populations? Australian Zoologist, 30: 316-324.

Zacchei D., Battisti C. \& Carpaneto G. M., 2011 - Contrasting effects of water stress on wetland-obligated birds in a semi-natural Mediterranean wetland. Lakes \& Reservoirs: Research and Management, 16: 281-286.

Zapparoli M., 2006 - Chilopodi della Maremma laziale e considerazioni sulla fauna del Monumento naturale "Palude di Torre Flavia". In: Biodiversità, gestione, conservazione di un'area umida del litorale tirrenico. Battisti C. (ed.). Provincia di Roma, Assessorato alle politiche agricole e dell 'ambiente, Gangemi editore, Roma: 217-229.

Zilli A., 2006 - Osservazioni sui Macroeteroceri (Lepidoptera). In: Biodiversità, gestione, conservazione di un'area umida del litorale tirrenico. Battisti C. (ed.). Provincia di Roma, Assessorato alle politiche agricole e dell'ambiente, Gangemi editore, Roma: 215-216. 